\title{
Formation en Nanosciences et Nanotechnologies : Un pas vers une «vraie» interdisciplinarité
}

\author{
H. Sellier ${ }^{1,3, a}$, E. Planus $^{2, b}$, F. Dubois ${ }^{2, a}$, L. Lévy ${ }^{1, a}$, I. Gautier-Luneau ${ }^{2, a}$, Ph. Peyla $^{1, c}$, F. \\ Marchi $^{1,3, a}$ \\ ${ }^{1}$ UFR PhITEM de l'Université Joseph Fourier, Grenoble 1 \\ ${ }^{2}$ UFR de Chimie et de Biologie de l'Université Joseph Fourier, Grenoble 1 \\ ${ }^{3}$ CIME Nanotech, 3 parvis Louis Néel, BP 257, 38016 Grenoble cedex 1 \\ ${ }^{a}$ Enseignant-chercheur à l'UJF et rattaché(e) à l'Institut Néel du CNRS \\ ${ }^{\mathrm{b}}$ Enseignante-chercheuse à l'UJF et rattachée à l'Institut Albert Bonniot (IAB - Inserm/UJF) \\ ${ }^{\mathrm{c}}$ Enseignant-chercheur à l'UJF et rattaché au Laboratoire Interdisciplinaire de Physique
}

Résumé : En 2011, l’Université Joseph Fourier (UJF), s'appuyant sur la force de la recherche interdisciplinaire des laboratoires grenoblois dans les Nanosciences et Nanotechnologies, a ouvert deux majeures : un M1 « Physique fondamentale et Nanosciences » et un M1 «Chimie et Nanosciences ». Dans ce cadre, des Travaux Pratiques interdisciplinaires et interplateformes ont été mis en place afin d'illustrer de manière concrète la convergence de la physique, de la chimie et de la biologie à l'échelle micro/nanométrique qui s'appuie sur les micro et nanotechnologies. Cet article décrit en détail ces TP et expose leur intérêt pédagogique pour la formation des étudiants ainsi que pour des enseignants-chercheurs.

Mots clés : Nanosciences, Nano(bio)technologies, Nanoparticules, Microscopie à Force Atomique, Microscopie Electronique, Microscopie à épi-fluorescence, Culture cellulaire, Cytosquelette, Micropattern, Nanomanipulation, retour d'expérience

\section{Introduction}

Depuis plusieurs années l'Université Joseph Fourier (UJF) propose une mention «Nanosciences et nanotechnologies » de Master en $2^{\text {ème }}$ année [1] en filières Recherche (Nanophysique, Nanochimie et Nanobiotechnologie) ou Professionnelle (Nanoingéniérie). En s'appuyant sur la force de la recherche interdisciplinaire des laboratoires grenoblois dans les Nanosciences et Nanotechnologies, l'UJF a depuis 2011, date de début du nouveau contrat quinquennal, renforcé sa formation dans ce domaine. Ont donc été ouvertes deux majeures «Nano» : un M1 «Physique fondamentale et Nanosciences» destiné aux étudiants physiciens et un M1 «Chimie et Nanosciences» destiné aux étudiants physico-chimistes, chimistes et biochimistes. L'ensemble des effectifs des deux parcours s'élève à 24 étudiants à ce jour.

Dans le cadre de ces deux nouveaux Master 1, des Travaux Pratiques interdisciplinaires et inter-plateformes ont été mis en place afin d'illustrer de manière concrète la convergence de la physique, de la chimie et de la biologie à l'échelle micro/nanométrique qui s'appuie sur les micro et nanotechnologies.

Ces séries de TP (une vingtaine d'heures au total) s'intègrent dans une UE spécifique de 50h ou 72h (6ECTS ou 9ECTS) intitulée "Nanosciences » dont la partie cours est déclinée en deux versions, l'une pour les physiciens, l'autre pour les (bio)chimistes. Elles s'articulent autour des thématiques de Nanochimie, Nano-caractérisation et Nanobiophysique ; Ces séries de TP ainsi que les cours associés, sont assurés par des enseignants-chercheurs de chimie, biologie et physique spécialistes en nanosciences et nanotechnologies.

Une première session de $8 \mathrm{~h}$ ou $12 \mathrm{~h}$ (deux ou trois séances de $4 \mathrm{~h}$ de TP) porte sur la «synthèse de nanoparticules métalliques et leur nano-caractérisation par MEB et AFM». La partie 
synthèse se déroule sur le plateau de Chimie situé au campus de l'UJF tandis que la nanocaractérisation a lieu au CIME-Nanotech [2] -Centre Interuniversitaire de MicroElectronique et de Nanotechnologie- (Salle Blanche et Nanomonde) situé à Minatec et ou à l'Institut Néel pour la caractérisation par Microscopie Electronique à Balayage (MEB).

Une deuxième session de $12 \mathrm{~h}$ (trois séances de $4 \mathrm{~h}$ de TP) porte sur la «Présentation de cellules animales sur substrats micro-fonctionnalisés pour leur caractérisation mécanostructurale par microscopie de fluorescence et par AFM». Cette série se déroule d'une part sur la plateforme CUBE [3] (Centre Universitaire de Biologie Expérimentale) du campus pour la partie biologie cellulaire et pour la caractérisation des cellules par épifluorescence, d'autre part sur la plateforme Nanomonde du CIME-Nanotech pour la nano-caractérisation par AFM des substrats micro-fonctionnalisés puis pour l'étude morphologique des cellules adhérentes. La complémentarité de ces deux techniques de caractérisation (épifluorescence et AFM) est soulignée lors des séances.

\section{Description de la session «Nanochimie et Nano-caractérisation»}

Cette session concerne l'élaboration de nanoparticules métalliques par réduction chimique de sels métalliques et la caractérisation par spectroscopie et microscopie MEB et/ou AFM des objets obtenus.

Nous avons choisi la thématique des nanoparticules car en raison de la taille de ces objets, des propriétés nouvelles sont attendues liées au confinement quantique et au grand rapport surface/volume. La maitrise de la taille de ces particules a permis de faire émerger de nouvelles applications en nanoélectronique [4], en optoélectronique [5] et dans la détection chimique ou biochimique [6]. Dans le cadre de ces travaux pratiques, nous proposons aux étudiants, lors d'une première séance, de synthétiser des suspensions colloïdales de nanoparticules d'or et d'argent en faisant varier un des paramètres de synthèse afin d'observer leur influence sur la taille ou la distribution en taille des objets obtenus. Lors d'une seconde séance, des échantillons sont observés au microscope électronique à balayage et/ou à l'AFM afin de consolider les études sur la forme, la dimension et la distribution en taille des échantillons. Grâce à la capacité de l'AFM à manipuler les objets, on pourra mettre aussi en évidence l'état d'adhésion des nanoparticules avec le substrat.

\subsection{Organisation de la session}

Ces Travaux Pratiques permettent de montrer aux étudiants un exemple d'interdisciplinarité à l'interface physique/chimie qui est particulièrement importante en Nanosciences. Même si la première partie est orientée vers la chimie avec la synthèse des particules et ensuite le reste de la session est plutôt orienté physique avec la caractérisation des objets synthétisées, les notions de « chimie » et de " physique » sont nécessaires à chaque étape pour mettre au point les protocoles et interpréter les observations.

Cette session comporte 5 phases que nous avons organisées en 3 séances de TP :

- $\quad$ Synthèse chimique $=\mathrm{TP}^{\circ} 1$

- $\quad$ Spectroscopie optique $=\mathrm{TP} n^{\circ} 1$

- Transfert sur graphite $=$ réalisé par les enseignants avant le TP $\mathrm{n}^{\circ} 2$

- Microscopie électronique $=\mathrm{TP} \mathrm{n}^{\circ} 2$

- $\quad$ Microscopie à force atomique $=\mathrm{TP} \mathrm{n}^{\circ} 3$

Cette répartition peut être adaptée en fonction du matériel disponible sur les différentes plateformes de TP et certaines séances sont indépendantes (TP $n^{\circ} 2$ et $T P n^{\circ} 3$ ), ce qui permet de moduler la longueur de cette session et de l'adapter à un nombre fixé de séances selon les filières. 


\subsection{Synthèse et spectroscopie des nanoparticules}

Cette séance, présentée dans cette partie, permet de sensibiliser les étudiants à la synthèse de nanoparticules, à l'influence des paramètres de synthèse sur la dimension et la distribution en taille des objets nanométriques obtenus et à la notion de stabilité de colloïdes en suspension dans un liquide. Toutes les expériences se font dans l'eau, elles sont faciles à mettre en place et sont modulables aisément.

La synthèse de colloïdes stables de nanoparticules métalliques peut se faire par une approche bottom-up, via la réduction d'un sel métallique, en présence d'agent stabilisant. La taille des nanoparticules obtenues, la distribution en taille ou la stabilité colloïdale dépendent fortement des conditions de réaction ( $\mathrm{pH}$, type de sel métallique...), de la force de l'agent réducteur et/ou du rapport de concentration [agent stabilisant]/[Métal]. Les suspensions colloïdales sont analysées par spectroscopie d'absorption, donnant une première estimation de la dimension des particules formées.

\subsubsection{Synthèse de nanoparticules d'or}

Les nanoparticules d'or sont obtenues, via la méthode, très connue dans la communauté scientifique, de Turkevich [7], Cette méthode se base sur la réduction de l'acide chloroaurique $\left(\mathrm{HAuCl}_{4}\right)$ en présence de citrate de sodium à reflux de l'eau en 30 minutes.

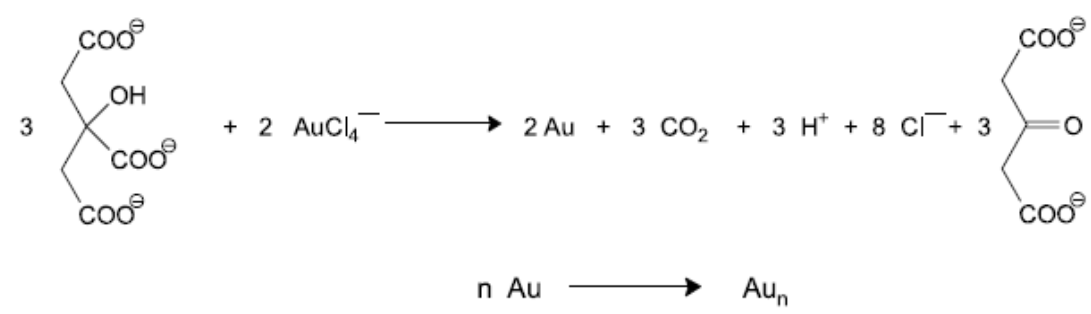

Le citrate de sodium joue dans ce cas à la fois le rôle d'agent réducteur et d'agent stabilisant permettant l'obtention de suspensions de colloïdes stables. Le rapport entre la concentration en citrate de sodium et la concentration en or est le point clé pour le contrôle de la taille des colloïdes obtenus par cette méthode. Au plus ce rapport [citrate]/[Au] est grand, au plus les nanoparticules sont petites. Cela s'explique par la compétition entre le processus de nucléation croissance de l' $\mathrm{Au}^{0}$ et le processus de passivation de la surface des nanoparticules d'or par le citrate de sodium. Deux expériences sont donc réalisées en faisant varier ce rapport de concentration [citrate]/[Au] d'un facteur 2, de 3.5 à 7 .

Une première approche pour caractériser la taille des nanoparticules synthétisées est de relever le spectre d'absorption de la suspension colloïdale obtenue. En effet, les suspensions colloïdales de nanoparticules d'Au présentent une coloration rouge-rubis lorsque la taille des nanoparticules est de l'ordre de la dizaine de nanomètres (figure 1A). Cette couleur, caractéristique des colloïdes nanométriques d'or est la conséquence, immédiatement visible, du confinement dans les nanoparticules de l'onde électromagnétique qui décrit la lumière (résonance de plasmon localisée). La coloration particulière des nanoparticules, liée à ce phénomène de résonance de plasmon localisée, se traduit pour les nanoparticules nanométriques d'or par une absorption de la lumière autour de $520 \mathrm{~nm}$ (figure 1B). La position de cette bande d'absorption dépend cependant d'un certain nombre de paramètres tels que le diamètre, la forme des nanoparticules, le milieu dispersif ou l'agrégation de la suspension colloïdale. 

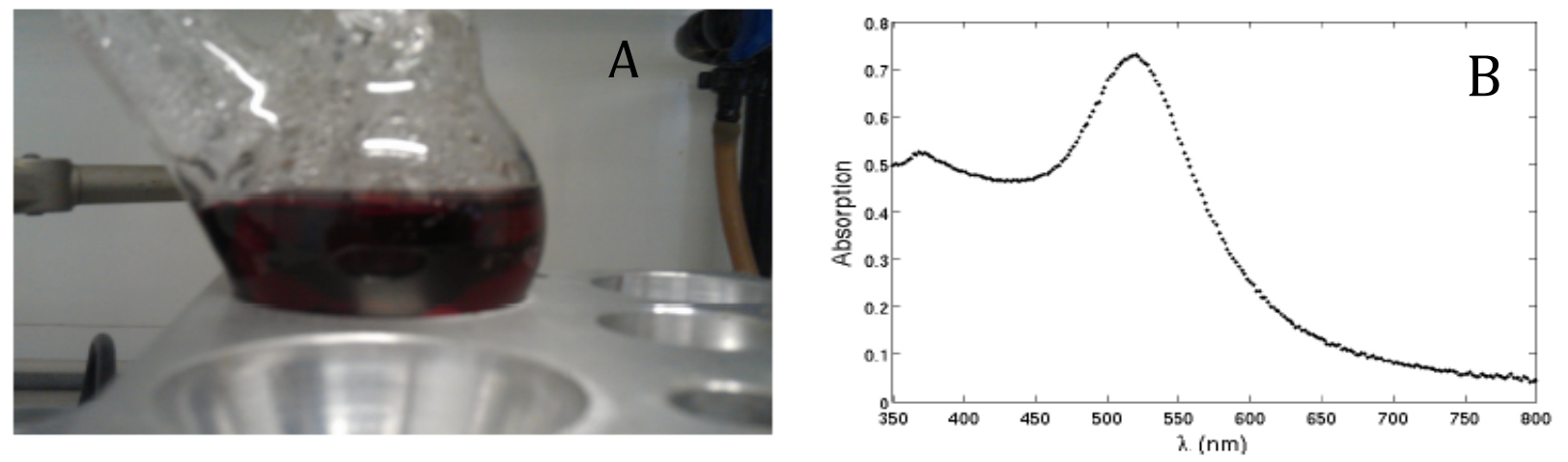

Figure 1: A) image de la suspension collö̈dale de nanoparticules d'Au obtenue par un étudiant avec un rapport $[$ citrate]/[Au] $=3.5$ après 30 minutes de réaction. $\mathrm{B}$ ) spectre d'absorbance correspondant, indiquant la présence de nanoparticules de diamètre d'environ $20 \mathrm{~nm}$.

Une comparaison entre les spectres d'absorbance obtenus par les étudiants et les données de la littérature indique que les nanoparticules obtenues avec un rapport [citrate]/[Au] $=3.5$ ont des tailles variant de 15 à $25 \mathrm{~nm}$. De plus, nous observons bien une diminution moyenne de la taille des particules lorsque la concentration en citrate de sodium augmente (taille comprise entre 10 et $20 \mathrm{~nm}$, en fonction des binômes d'étudiant). La faible largeur à mi-hauteur des spectres d'absorbance indique que cette méthode de synthèse est relativement peu polydisperse.

\subsubsection{Synthèse de nanoparticules d'Argent}

Les synthèses de nanoparticules d'argent sont réalisées par réduction du nitrate d'argent par l'acide ascorbique $\left(\mathrm{C}_{6} \mathrm{H}_{8} \mathrm{O}_{6}\right)$ en présence de citrate de sodium [8]. L'acide ascorbique joue le rôle d'agent réducteur et le citrate de sodium celui d'agent stabilisant. Dans ce système, la taille des nanoparticules dépend grandement des conditions de synthèse. Ainsi, pour des concentrations en agent stabilisant et en agent réducteur identiques, la taille des nanoparticules obtenues peut être modulée en modifiant le $\mathrm{pH}$ de la solution. L'augmentation du $\mathrm{pH}$ jouant le rôle d'activateur de l'agent réducteur et donc modifie la cinétique de formation des nanoparticules.

$$
2 \mathrm{AgNO}_{3}+\mathrm{C}_{6} \mathrm{H}_{8} \mathrm{O}_{6} \rightarrow 2 \mathrm{Ag}+\mathrm{C}_{6} \mathrm{H}_{6} \mathrm{O}_{6}+2 \mathrm{HNO}_{3}
$$

Dans le cadre du TP, deux synthèses sont donc réalisées, dans les mêmes conditions de concentration en nitrate d'argent, en acide ascorbique et en citrate de sodium, mais à $\mathrm{pH}$ différents $(\mathrm{pH}=6,3$ et $\mathrm{pH}=10,5)$. Les réactions se font dans l'eau, à température ambiante et le $\mathrm{pH}$ des solutions est fixé par l'addition de $\mathrm{NaOH} 0,1 \mathrm{~mol} / \mathrm{L}$.

Dans le cas des nanoparticules d'argent, le phénomène de résonance de plasmon localisée provoque l'apparition d'une bande d'absorption présentant un maximum entre 390 et $420 \mathrm{~nm}$ en fonction de la dimension des particules. Une analyse du spectre d'absorption donne donc des indications directes sur la taille et la distribution en taille des nanoparticules (figure 2).

La formation des nanoparticules d'argent à pH 6.3 est lente et engendre des particules de grandes tailles (35 à $50 \mathrm{~nm}$ voir plus) et est très polydisperse en raison de la faible activité chimique de l'agent réducteur dans ces conditions de $\mathrm{pH}$. Par contre, comme le montre le spectre d'absorption, la formation des particules à $\mathrm{pH}$ basique $(\mathrm{pH}=10.5)$ permet l'obtention rapide de plus petites particules, et relativement monodisperses (spectre d'absorbance étroit). Le maximum d'absorption obtenue pour ces particules est situé vers $395 \mathrm{~nm}$ correspondant à des diamètres de particules compris entre 10 et $15 \mathrm{~nm}$. 


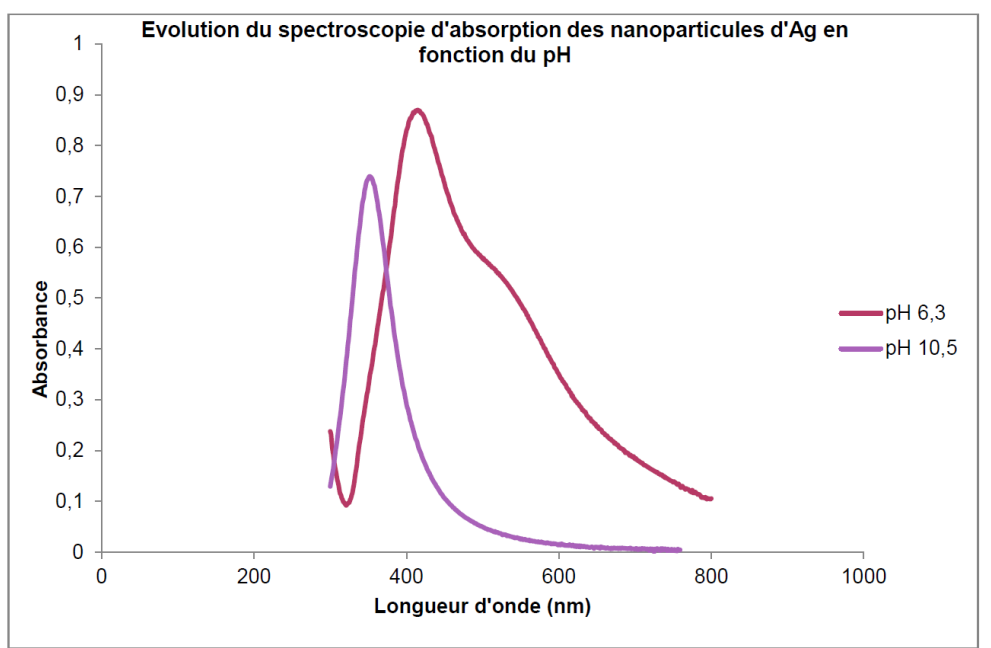

Figure 2 : Spectre d'absorbance des suspensions colloïdales de nanoparticules d'argent obtenues par réduction du nitrate d'argent par l'acide ascorbique à deux $\mathrm{pH}$ différents $(6,3$ et 10,5)

La synthèse et la caractérisation UV-visible des échantillons se fait sur la plateforme de chimie analytique (PCA) de l'UFR de chimie et de biologie de Grenoble [9]. Cette plateforme dispose de salles de chimie équipées de Sorbonne et d'une salle d'analyse proposant une très grande partie des techniques d'analyse du domaine de la chimie analytique (chromatographie, spectrométrie de masse, spectrophotométrie (UV, IR, fluorescence), DSC, rhéologie, polarimétrie...

\subsection{Préparation des échantillons pour la microscopie}

La suite de cette session a pour objectif de visualiser les nanoparticules synthétisées par microscopie électronique (MEB) et par microscopie de force à sonde locale (AFM), afin de déterminer leur forme, de mesurer leur taille, et même de les manipuler. Pour cela, il est nécessaire de transférer les nanoparticules depuis la suspension colloïdale vers un substrat solide. La surface choisie pour déposer les nanoparticules est une surface de carbone graphite HOPG (Highly Ordered Pyrolytic Graphite) car elle est plate à l'échelle atomique sur plusieurs centaines de nanomètres, formant ainsi de larges terrasses délimitées par des marches dont l'épaisseur est de quelques plans atomiques. Outre la bonne planéité de ce substrat, le carbone permet d'obtenir un très bon contraste avec les particules d'or et d'argent en microscopie électronique. En revanche, la très faible physisorption des particules sur la surface de graphite nécessite d'opérer la microscopie à force atomique en mode oscillant pour ne pas déplacer les particules pendant le balayage de la sonde.

Dans un premier temps, un échantillon carré de graphite HOPG de $5 \mathrm{~mm}$ de côté est collé avec de la laque d'argent sur un disque métallique d'un centimètre de diamètre pour servir de support compatible avec les microscopes MEB et AFM. Le collage doit être conducteur pour évacuer les charges lors de la microscopie électronique. Dans un second temps, les nanoparticules initialement en solution sont déposées sur la surface de graphite par centrifugation à l'aide d'un "spin-coater» (tournette). L'échantillon de graphite est fixé au centre de la platine tournante et une goutte $(10 \mu \mathrm{L})$ de solution est déposée sur le graphite à l'aide d'une micropipette. La platine est mise en rotation pendant 30 secondes à une vitesse de 1000 tours par minute $(\mathrm{rpm})$ pour éjecter le liquide par centrifugation et laisser seulement quelques particules sur la surface (environ 1 à 10 par $\mu \mathrm{m}^{2}$ ). Dans certains cas les nanoparticules s'agglutinent par petits paquets, alors que dans d'autres cas elles restent bien séparées les unes des autres.

Cette préparation des échantillons pour la microscopie n'a pas été intégrée dans les séances de travaux pratiques principalement pour des raisons d'absence de cet équipement sur les 
plateformes d'enseignement. Elle est donc effectuée par les enseignants à l'institut Néel en utilisant les solutions de nanoparticules obtenues par les étudiants. Plusieurs échantillons ont été préparés avec des particules d'or ou d'argent et des conditions de synthèse différentes en terme de $\mathrm{pH}$ ou de concentration en citrate de sodium. Chaque échantillon a été numéroté pour pouvoir l'identifier par la suite et permettre aux étudiants de corréler les observations de microscopie avec les conditions de synthèse.

\subsection{Caractérisation des nanoparticules par microscopie électronique à balayage (MEB)}

Au début de la séance, l'enseignant commence par décrire le fonctionnement du microscope, les réglages du faisceau, et les différents détecteurs d'électrons utilisés pour l'imagerie MEB, en se basant sur un échantillon de test pour effectuer les explications. Puis il place un ou plusieurs échantillons avec les nanoparticules dans la chambre d'observation du microscope (manipulation délicate). Ensuite chacun des étudiants du groupe prend les commandes du MEB et a pour mission d'analyser un des échantillons de graphite sur lesquels sont déposés les nanoparticules d'or ou d'argent. L'étudiant affine les réglages et fait des images de la surface à différents grossissements pour visualiser les nanoparticules, leur forme, leur dispersion, et leur positionnement par rapport aux terrasses de graphite. Il mesure ensuite la taille de quelques particules et les compare aux résultats de spectroscopie optique d'absorption faite à la première séance. Les images de la figure 3 prises par les étudiants montrent que les particules d'or sont toujours bien sphériques et relativement mono-disperses avec un diamètre autour de $15 \mathrm{~nm}$. En revanche les particules d'argent sont d'une forme plus complexe et leur taille varie entre 10 à $100 \mathrm{~nm}$. Ces observations sont en bon accord avec les résultats de spectroscopie optique. Les microscopes électroniques utilisés sont des «Zeiss Ultra + » (CIME-Nanotech et Institut Néel).
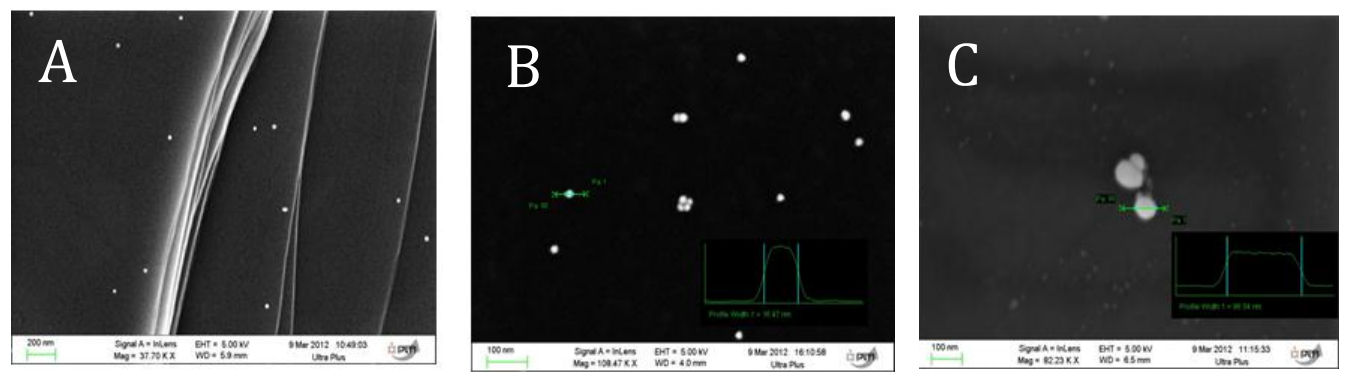

Figure 3 : Images MEB des nanoparticules déposées sur graphite (images prises par les étudiants) : (A) Marches du graphite avec nanoparticules d'or sur les terrasses. (B) Nanoparticules d'or (taille mesurée = $16 \mathrm{~nm}$ ). (C) Nanoparticules d'argent (taille mesurée $=66 \mathrm{~nm}$ ).

\subsection{Caractérisation par microscopie à force atomique (AFM) des nanoparticules}

$\mathrm{Au}$ cours de cette séance, les étudiants apprennent à faire fonctionner un microscope AFM depuis la mise en place de la sonde de force jusqu'à l'obtention et l'analyse des images, en passant par les réglages de la détection optique et des paramètres d'asservissement du système.

La première partie du TP consiste à étudier la surface de l'échantillon en mode "tapping » dans lequel le contact de la pointe est intermittent grâce à la vibration du levier excité à sa fréquence de résonance. En changeant la taille et la vitesse de balayage, les étudiants peuvent observer l'arrangement des nanoparticules dispersées aléatoirement sur la surface et mesurent le profil topographique de quelques particules pour déterminer leur taille. Les marches atomiques de la surface de graphite sont également bien visibles et il est possible de mesurer leur hauteur avec une résolution meilleure que le nanomètre. 

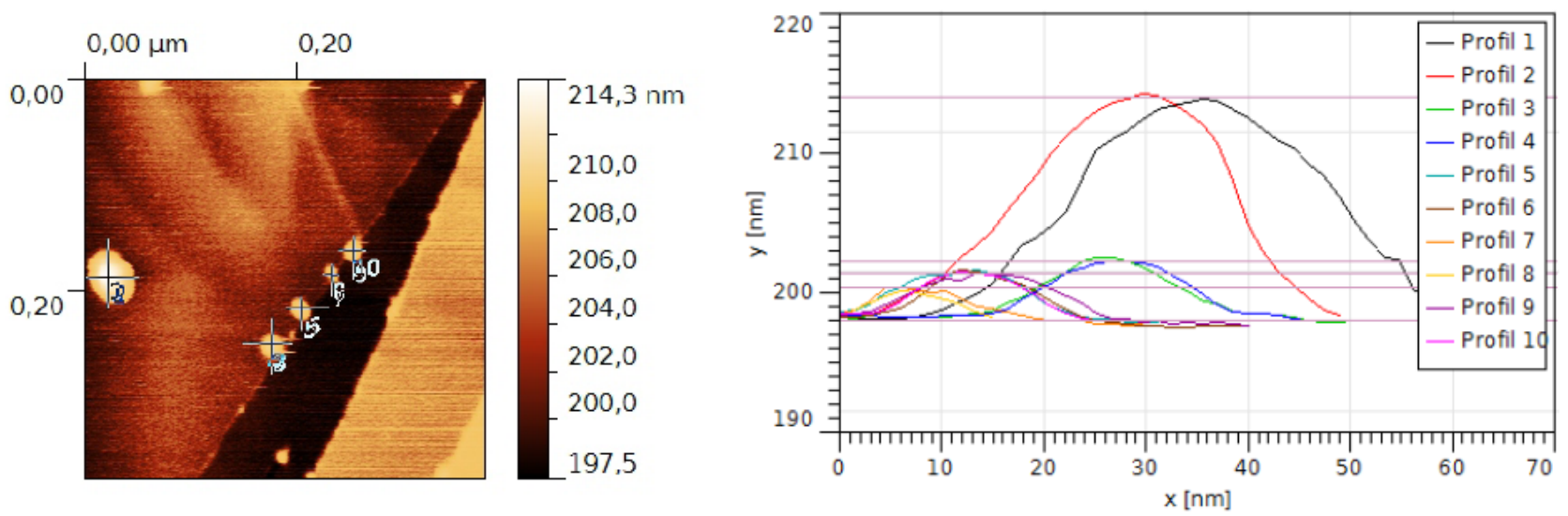

Figure 4 : Cartographie AFM des nanoparticules d'or déposées sur la surface de graphite et analyse des profils topographiques de cinq particules (extrait d'un compte-rendu d'étudiant).

La figure 4 montre une analyse de taille de particules d'or obtenue grâce au logiciel gratuit de traitement d'images "Gwyddion» et tirée d'un compte-rendu de TP. Sur l'image topographique de gauche, on y voit plusieurs terrasses du graphite de hauteur différente et quelques particules posées sur ces terrasses. Les étudiants ont analysé les dimensions de cinq particules en traçant des profils topographiques sur la figure de droite. La particule la plus à gauche a ainsi un diamètre de $16 \mathrm{~nm}$, donné par la hauteur du profil, et non par la largeur qui est de $30 \mathrm{~nm}$ car cette dernière résulte de l'effet de convolution entre la forme de la particule et la forme de la pointe du microscope AFM (les particules d'or sont normalement sphériques). La comparaison des images MEB et AFM est de ce point de vue très instructive, car le type de sonde est très différent (faisceau d'électron versus pointe silicium gravée) et le phénomène de convolution est plus faible avec le MEB (si les réglages sont faits correctement). Ces deux microscopies à résolution nanométrique sont donc très complémentaires pour l'étude des nano-objets.

Ce diamètre de $16 \mathrm{~nm}$ correspond bien aux résultats de caractérisation par spectrométrie optique faite lors du TP de synthèse par voie chimique. L'image montre également quatre particules beaucoup plus petites, de diamètre entre 2 et $4 \mathrm{~nm}$, positionnées le long d'une marche de la surface de graphite. Il existe donc des particules d'or dont la nucléation a été stoppée plus vite que la moyenne.

La deuxième partie du TP consiste à mettre en évidence la faible adhésion des particules sur le graphite en changeant le mode d'imagerie dynamique (figure 5A) pour un mode « contact» statique, avec une très faible force d'appui exercée sur la pointe, mais qui suffit pour pousser les particules hors de la zone balayée (figure 5B). Les particules sont ainsi déplacées par la pointe sur le pourtour de l'image, comme le montre les images faites par la suite en mode dynamique (figure 5C). Cette opération est une démonstration des possibilités de manipulation de nano-objets par les microscopes à sondes locales en champ proche comme l'AFM et le STM (microscope à effet tunnel). 
A

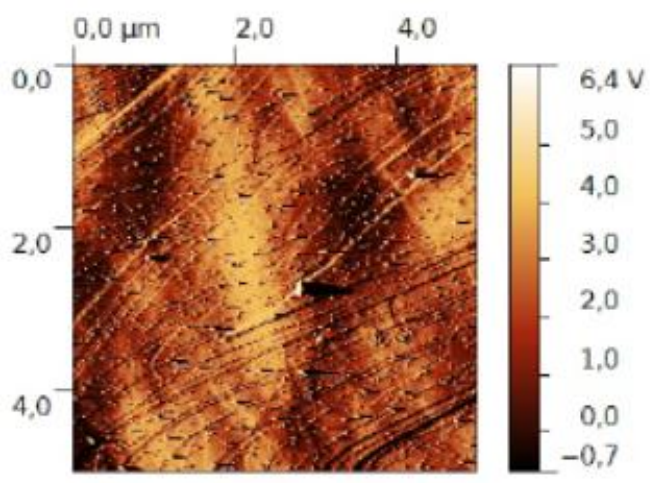

B

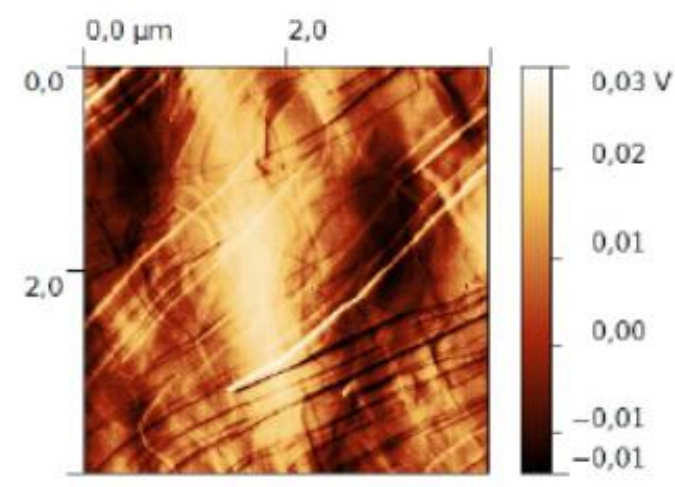

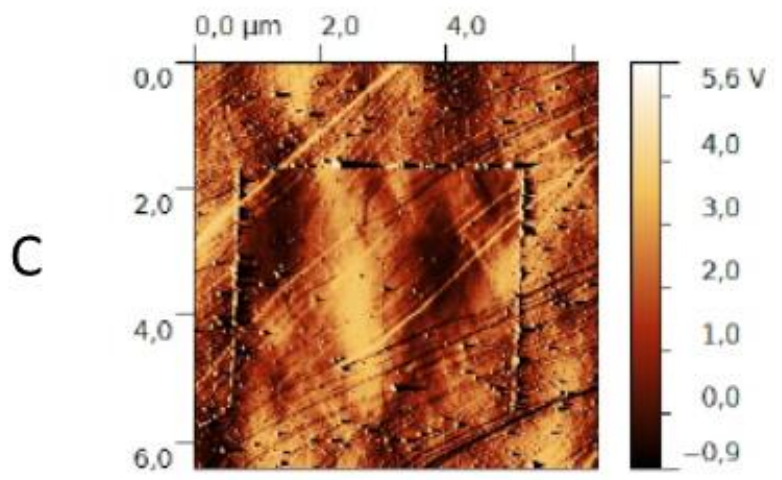

Figure 5 : Démonstration du déplacement des nanoparticules par un balayage de la pointe en mode «contact» (extrait d'un compte-rendu d'étudiant) : (A) surface initiale imagée en mode «tapping" sur 5 x $5 \mu^{2}$ (carte de variation d'amplitude). (B) surface pendant le balayage en mode "contact» sur 4 x $4 \mu \mathrm{m}^{2}$ (carte de déflexion verticale). (C) surface finale imagée en mode "tapping" sur 6 x $6 \mu \mathrm{m}^{2}$ (carte de variation d'amplitude). Les nanoparticules initialement présentes ne sont plus visibles en mode «contact» car elles sont poussées par la pointe pendant le balayage comme le montre l'image finale en mode «tapping " avec un alignement de particules sur le pourtour carré de la zone balayée en mode « contact».

Ce TP complet illustre à travers un exemple concret l'aspect physico-chimique des Nanosciences. Comme cette UE «Nanosciences» du master 1 Nano peut aider les étudiants à choisir leur orientation en M2 Nano soit vers le parcours nanophysique ou nanochimie ou nanobiotechnologies, nous avons jugé indispensable de monter une deuxième série de TP autour de l'interface biologie et physique que nous avons dénommée session «Nanobiophysique».

\section{Description de la session « NanoBioPhysique »}

Des recherches récentes ont montrés le lien entre propriétés physique, mécanique et propriétés physiologique des cellules animales. Par exemples, l'augmentation de la valeur du module de Young entre une cellule saine et une cellule cancéreuse [10], le rôle de la pression sur certaines phases du développement de l'embryon de drosophile $[11,12]$ ou encore la conception du tri cellulaire basé sur des micro-aimants phagocytés par les cellules [13]. Au cœur de toutes ces études et leurs applications potentielles se trouve un travail pluridisciplinaire, emblématique des Nanosciences et Nanotechnologies.

Cette série de TP a pour but d'illustrer à la fois la convergence des techniques d'élaboration et de caractérisation ainsi que la démarche scientifique expérimentale entre la biologie et la physique à l'échelle nanométrique. 
Pour atteindre cet objectif pédagogique, nous avons conçu une série de trois $\mathrm{TP}$ associée à $7 \mathrm{~h}$ de cours assurés par deux enseignantes-chercheuses, une physicienne spécialiste en microscopie en champ proche et une biologiste spécialiste en biomécanique de la cellule. La thématique choisie est l'étude des mécanismes d'adhésion et de migration de cellules animales sur un substrat micro-bio-fonctionnalisé suivie de la caractérisation de leurs propriétés morphologiques et mécaniques.

Les cellules des tissus sont habituellement en contact avec un réseau complexe de macromolécules sécrétées par la cellule elle-même. Ce réseau de polymères enchevêtrés se défini comme la matrice extracellulaire. L'ensemble des interactions mécaniques et physiques entre la cellule et la matrice extracellulaire sont dues à des protéines transmembranaires spécifiques (en premier lieu les intégrines) qui jouent le rôle de molécules d'adhérence et qui sont aussi des mécanorécepteurs sensibles à aux propriétés mécaniques de l'environnement[14]. Ces adhérences permettent à la cellule de maintenir une homéostasie mécanique avec son environnement, on comprend alors que l'adhésion joue un rôle crucial dans les processus cellulaires. En effet, ce n'est que lorsque les cellules adhèrent qu'elles peuvent ensuite répliquer leur $\mathrm{ADN}$, se diviser, ou bien se différencier. Comme leur appellation le suggère, les intégrines sont des molécules qui traversent de part en part la membrane plasmique des cellules. Ce sont des récepteurs bi-phasiques qui permettent de connecter les polymères intracellulaires (micro filaments d'actine) aux polymères extracellulaires (les composants fibrillaires de la matrice extracellulaire, la fibronectine par exemple). La connexion aux micro-filaments d'actine est essentielle à la maturation des adhérences qui n'existent qu'en réponse aux contraintes mécaniques appliquées de l'extérieur et/ou développées par la cellule [15]. Ainsi, les micro-filaments d'actine forment un squelette intracellulaire relié aux adhérences dont la principale fonction est son aptitude à exercer des forces sur l'environnement : le cytosquelette est à la fois le squelette et le muscle de la cellule et se défini en parti par ses propriétés rhéologiques viscoélastiques.

Nous avons choisi dans cette session Nanobiophysique de mettre en évidence l'impact de la taille et de la répartition spatiale de la matrice extracellulaire (fibronectine) sur la morphologie adoptée par la cellule animale pour y adhérer. Les propriétés élastiques des différents constituants de la cellule sont comparées.

Pour y parvenir nous avons sélectionné un échantillon commercial micro-bio-fonctionnalisé disponible auprès de l'entreprise CYTOO issue du CEA (Centre de l'Energie Atomique) de Grenoble [16].

L'échantillon CYTOO est constitué de dépôts localisés et de formes géométriques variées d'une protéine, la fibronectine (Fbn) qui favorise l'adhérence des cellules. Ces dépôts sont réalisés grâce à des techniques de microélectronique sur un substrat mince $(170 \mu \mathrm{m})$ de verre qui est un des supports les plus courants en biologie cellulaire car il est peu cher, optiquement transparent et disponible en dimensions variées.

Entre les motifs de fibronectine, une couche homogène de polymère (PEG Polyethylene Glycol) est présente dont le caractère hautement hydrophobe est peu favorable à l'adhérence de cellules animales. Ainsi l'étalement des cellules sera confinée sur les zones de Fbn.

Afin d'obtenir des motifs de Fbn de formes et de position contrôlées à l'échelle micronique, leur fabrication fait appel aux techniques de lithographie et de dépôt développées depuis très longtemps en microélectronique. La première étape consiste en un traitement cytophobique à savoir qu'une couche de polymère de type PEG d'épaisseur sub-micrométrique est déposée en couche mince sur une lame de verre. Puis à travers un masque de chrome présentant des motifs de taille micrométrique et de formes géométriques variées, le polymère est éliminé par photo-ablation, laissant apparaitre ainsi des zones dans la couche de polymère dont la forme reproduit celle des motifs du masque (figure 6). La dernière étape repose sur un dépôt localisé en phase liquide de Fbn sur les motifs préalablement abrasés dans la couche de polymère. On 
obtient donc ainsi une collection de motifs de Fbn de formes géométriques et de tailles variables séparées par un polymère cytophobique.
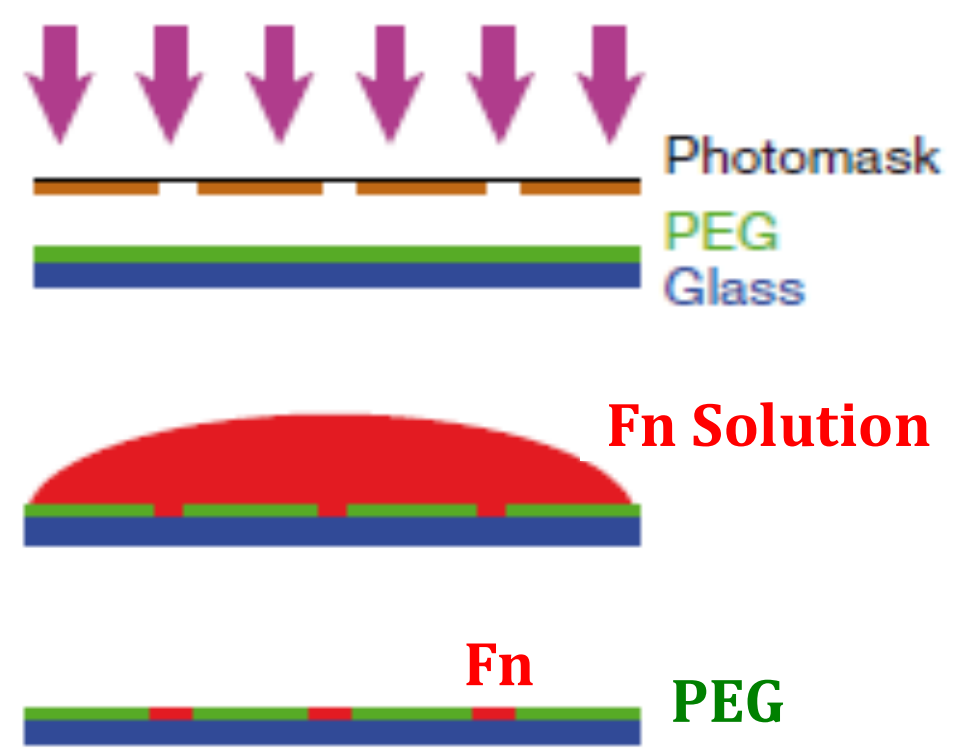

Figure 6: Principe des trois étapes majeures de fabrication de l'échantillon CYTOOchips ${ }^{\mathrm{TM}}$. Photo-lithographie du polymère PEG à travers un masque de chrome (haut), dépôt en phase liquide de fibronectine (milieu), obtention d'une collection de motifs de Fbn au sein du polymère anti-adhésif (bas).

Comme la faible épaisseur de la couche de Fn et de polymère rend invisible en microscopie optique les motifs, un système de coordonnées en colonnes et lignes labélisées respectivement par des chiffres et des lettres permet leur localisation. A chaque intersection de colonnes et de lignes correspond un carrée de $8 \mathrm{~mm}$ de côté contenant plusieurs micro-motifs identiques et disposés de manière régulière (figure 7). Le modèle "Starter's CYTOOchip » sélectionné pour le TP, présente à sa surface quatre formes de motifs de Fbn (cercle, arbalète, Y et I) en trois tailles différentes.

La procédure de fabrication de ces échantillons CYTOO est décrite en détail lors de la partie de nano-biochimie du cours associé à ces TP.

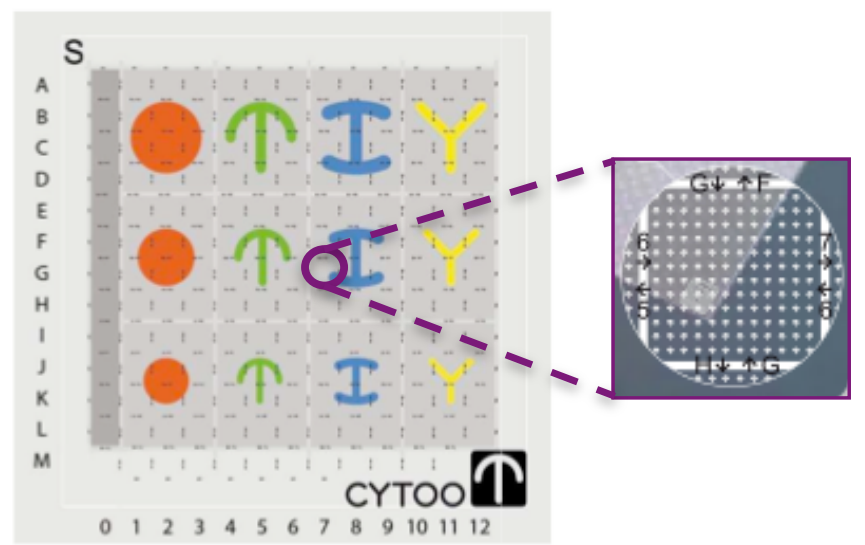

Figure 7: Représentation de l'échantillon « «Starter's CYTOOchip» avec ces motifs de Fbn, ces quatre formes géométriques et tailles différentes. Le système de repérage style «bataille navale » est présent sur les bords mais aussi à l'intérieur de l'échantillon (zoom à gauche). Chaque carré de $1 \mathrm{~mm}$ de côté contenant plusieurs motifs, est identifié par trois lettres et trois chiffres. 
Les trois séances de TP qui constituent cette session expérimentale de Nanobiophysique durent chacune $4 \mathrm{~h}$ et les thématiques abordées sont décrites en détails dans la suite de cette section :

- Caractérisation par AFM d'une surface micro-bio-fonctionnalisée et introduction à la spectroscopie de force (section 3.1) $=\mathrm{TP}^{\circ} 1$

- Analyse in cellula par microscopie à épi-fluorescence de l'adhérence et du squelette de cellules ostéoblastiques de souris structurées sur un patron adhésif de fibronectine (section 3.2) $=\mathrm{TP} \mathrm{n}^{\circ} 2$

- Etude comparée par AFM des propriétés morphologiques et mécaniques des cellules ostéoblastiques de souris adhérentes sur un patron adhésif de fibronectine $($ section 3.3$)=\mathrm{TP} \mathrm{n}^{\circ} 3$

\subsection{Caractérisation d'une surface micro-bio-fonctionnalisée par AFM et introduction à la spectroscopie de force (TP $\left.n^{\circ} 1\right)$}

L'objectif de cette première séance de TP de 4 h est triple pour les étudiants : (1) s'initier à la microscopie à force atomique (AFM), (2) l'utiliser pour caractériser l'échantillon micro-biofonctionnalisé modèle " Starter's CYTOOchip » qui servira lors de la séance suivante à faire adhérer les cellules animales et enfin (3) s'initier à la spectroscopie de force qui permet entre autre, de sonder les propriétés viscoélastiques des matériaux.

\subsubsection{Prise en main de l'AFM et caractérisation du pattern CYTOO ${ }^{T M}$}

En tout début de séance, les étudiants observent au microscope optique (LEICA, possédant un zoom optique réglable) l'échantillon de calibration en silicum (réseau périodique de trous de forme rectangulaire) ainsi que l'échantillon « Stater's CYTOOchip ».

L'observation au microscope optique du réseau permet de mesurer le pas du réseau et les dimensions du motif rectangulaire (figure 8a). L'image optique renseigne ainsi sur la taille de l'image à effectuer en AFM pour observer quatre à six motifs. A partir de l'image AFM les étudiants pourront mesurer la profondeur nanométrique des motifs, paramètre non accessible en microscopie optique. L'image optique de l'échantillon «Stater's CYTOOchip » permet d'une part de se familiariser avec le système de repérage et d'autre part de se rendre compte qu'il n'est pas possible de distinguer les micromotifs dans la couche de polymère (figure $8 \mathrm{~b}$ ). Ceci démontre que l'épaisseur du dépôt Fbn et de la couche de polymère sont très proche. Cette partie consacrée à l'observation optique dure environ $30 \mathrm{~min}$. Elle illustre le fort potentiel de la microscopie optique qui reste un instrument de choix pour les scientifiques tout en montrant ses limites. Les étudiants prennent donc bien conscience de la nécéssité d'utiliser d'autres techniques pour caractériser leurs échantillons à l'échelle nanométrique. Pour terminer cette partie introductive, les étudiants observent et enregistrent des images de sondes AFM «contact» puis « tapping » afin d'en mesurer leurs dimensions géométriques. Chaque binôme sauvegarde l'ensemble de leurs images sur une clés USB au format JPEG afin de traiter et analyser leurs données à la maison pour rédiger leur compte-rendu. Les étudiants sont donc à présent prêts pour aborder la partie du TP dédiée à la caractérisation morphologique par AFM de ces deux échantillons.

Les étudiants prennent en main un des deux microscopes AFM (modèles 3100 et ICON de chez Brucker) disponibles sur la plateforme Nanomonde. Ils imagent en mode dynamique (Tapping ${ }^{\mathrm{TM}}$ ) le réseau de calibration. La mise en place de la sonde AFM et de l'échantillon sur le microscope AFM est assurée par le technicien de la plateforme.

Les étudiants débutent le TP par la mesure de la courbe de résonance (l'amplitude d'oscillation de l'extrémité du levier en fonction de la fréquence d'excitation) afin de 
déterminer la fréquence de résonance $f_{0}$ de la sonde AFM et son facteur de qualité $Q$ (figure 9).
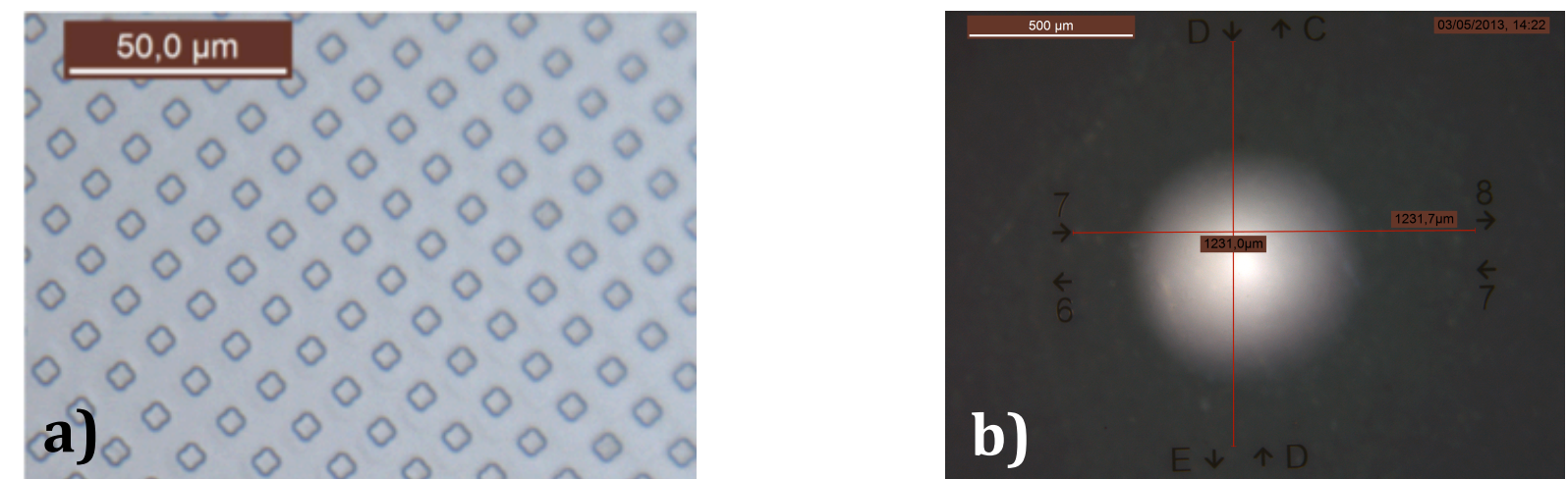

Figure 8 : images optiques issues des CR des étudiants. a) Réseau de calibration en silicium (gauche) où l'on observe les motifs rectangulaires sans pouvoir distinguer leur relief ; b) Surface de l'échantillon Stater's CYTOOchip où l'on peut distinguer les lettres et chiffres sur chaque carré indispensable pour la localisation des motifs de fibronectine.

A partir de la mesure de la fréquence de résonance et des mesures optiques de la longueur et largeur du levier, les étudiants estiment la constante de raideur de la sonde grâce à la relation suivant $(\mathrm{Eq} 1)$ :

$$
f_{0}=\frac{1}{2 \pi} \sqrt{\frac{k}{m^{*}}}
$$

La masse effective $m^{*}$ du levier ne dépend que de ses dimensions, de sa forme géométrique et de la densité volumique du matériau qui la constitue (généralement du silicium). La raideur du levier $k$ ne dépend que des dimensions du levier et du module de Young du matériau qui le constitue. Comme le seul paramètre dimensionnel qui n'est pas mesurable facilement par microscopie optique est l'épaisseur du levier, la mesure expérimentale de la fréquence de résonance permet donc de déterminer cette épaisseur et ainsi de calculer la valeur de $k$. La connaissance de la valeur de $k$ est indispensable pour l'interprétation des mesures de force qui seront menées dans le mode spectroscopie de force au cours du TP $\mathrm{n}^{\circ} 3$. On ne peut pas se contenter de la valeur moyenne indiquée sur la fiche technique de la sonde car la variation autour de cette valeur moyenne est d'un facteur 10 .

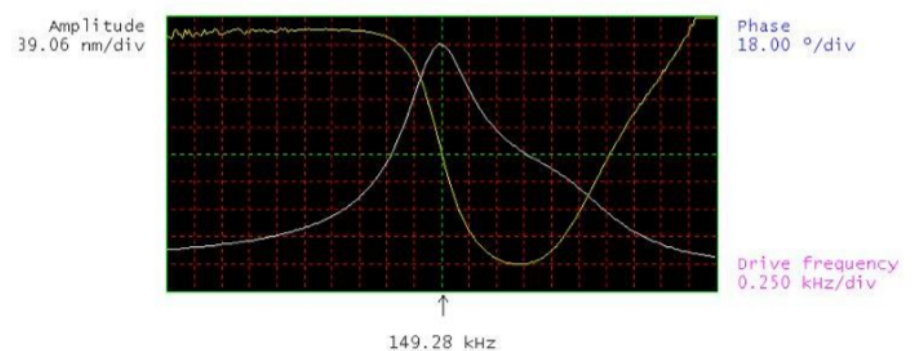

Figure 9 : Courbes de résonance de la sonde AFM, l'amplitude d'oscillation (blanc) et la phase (jaune) du levier en fonction de la fréquence d'excitation, $f_{0}=149,28 \mathrm{kHz}$

Une fois, la fréquence de résonance déterminée, la pointe est engagée sur la surface en mode dynamique et les étudiants ajustent la taille de l'image à partir de leurs observations optiques pour obtenir une image AFM comportant quelques motifs rectangulaires (figure 10A). Afin de comprendre le rôle de la boucle de contre-réaction et l'impact des valeurs de ces gains proportionnels et intégral sur la qualité et la reproductibilité de l'image, le balayage selon l'axe $\mathrm{Y}$ est coupé et la même ligne est balayée en permanence. Ainsi les étudiants peuvent observer directement l'impact des valeurs des gains sur la topographie mais aussi celle de la vitesse de balayage, du nombre de pixel ou encore du point de fonctionnement « setpoint » et 
déterminer ainsi la configuration adéquate pour obtenir une image reproductible et fiable (avec un bruit minimisé).

Une fois le fonctionnement de l'AFM en mode imagerie maîtrisé, les étudiants ré-investissent leur nouveau savoir-faire afin de caractériser l'échantillon micro-biofonctionnalisé.

Comme la taille maximale de balayage du microscope AFM est limitée à $100 \mu \mathrm{m}$, seuls les plus petits motifs de l'échantillon "Starter's CYTOOchip » peuvent être imagés en entier (Lignes I,J,K,L). Les étudiants choisissent la forme (arbalète, cercle, I, Y) du motif qu'ils désirent caractériser. A partir de l'image AFM, toutes les dimensions du motif sont mesurables, et on se rend compte que l'épaisseur du dépôt de Fbn est légèrement inférieure à celui de la couche de polymère car les motifs apparaissent en creux (figures 10B et 10C). La différence d'épaisseur est de l'ordre de $10 \mathrm{~nm}$, ce qui confirme que les épaisseurs des deux dépôts sont similaires et que la surface peut être considérée relativement plate du point de vue des cellules qui vont y adhérer. Cette observation est importante car le comportement des cellules peut-être affectés par la rugosité de la surface [17].

Deux heures sont nécessaires pour réaliser l'ensemble des cartographies AFM (réseau et surface CYTOO).

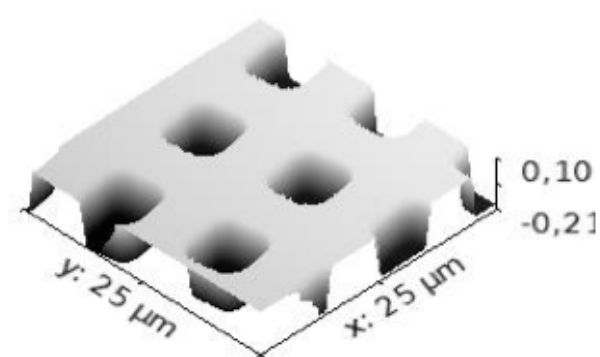

A)

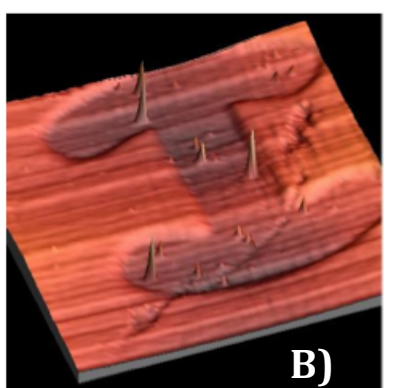

B)

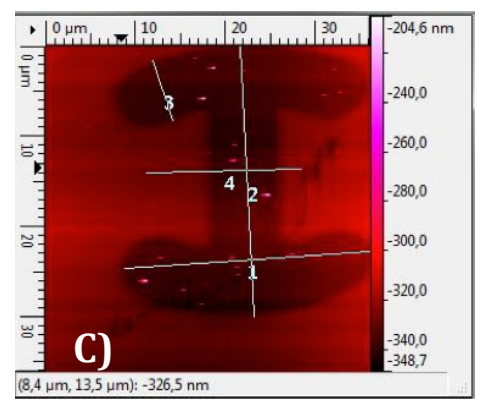

Figure 10 : A) images AFM en 3D du réseau de calibration dont on peut mesurer la hauteur du motif en creux (300nm environ), B) image AFM en 3D du motif CYTOO et C) image AFM en 2D d'un motif CYTOO ${ }^{\text {TM }}$ avec les mesures de dimensions latérales effectuées par les étudiants. Ces trois images sont issues de CR d'étudiants.

Comme l'AFM permet d'obtenir la morphologie d'échantillon avec une résolution nanométrique mais aussi de mesurer l'interaction pointe-surface en différents points de la surface de l'échantillon, son usage se répand de plus en plus pour étudier les propriétés mécaniques des échantillons. Le mode de base pour mener ces études est le mode spectroscopie de force.

\subsubsection{Initiation à la Spectroscopie de force grâce au Nanomanipulateur à retour de force}

Dans la deuxième partie de la séance les étudiants s'initient au mode spectroscopie de force durant 1 h30 grâce au nanomanipulateur à retour de force $[18,19]$ et plus spécifiquement la scène virtuelle en 1D (figure 11).

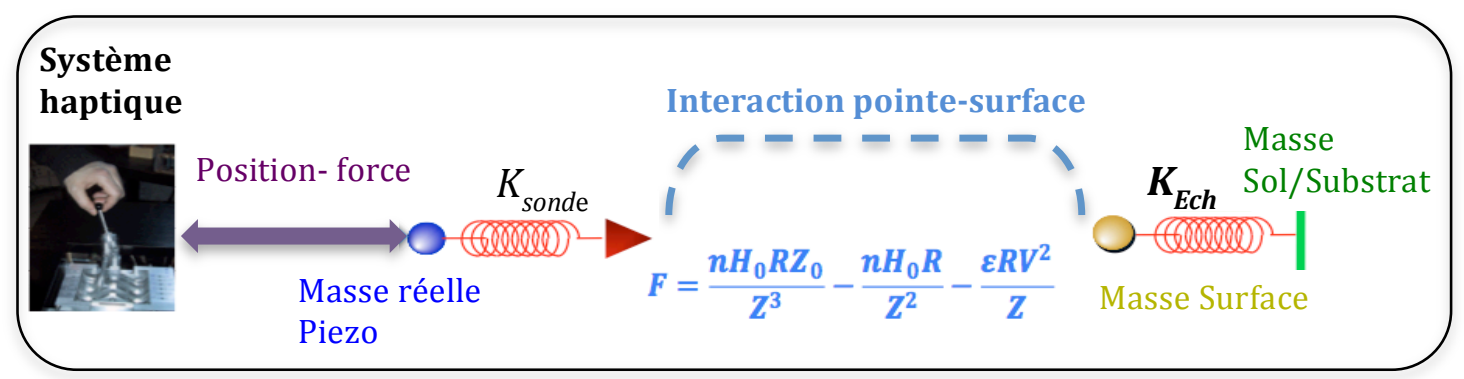

Figure 11 : schéma de principe de la scène virtuelle 1D du NanoLearner. F est la force totale entre la pointe et l'échantillon; trois termes constituent la force où $R$ est le rayon de la pointe AFM, $H_{0}$ la constante de Hamaker, $n$ un 

la distance entre la pointe et la surface.

Ils étudient l'impact de la valeur de la raideur de la sonde $\left(K_{\text {Sonde }}\right)$ et de l'élasticité de l'échantillon $\left(K_{E c h}\right)$ sur les courbes de force. Pour cela, ils pilotent à la main la sonde AFM pour effectuer des courbes de forces et enregistrent via le logiciel associé, les déformations de la sonde AFM lors de son interaction avec l'échantillon. Par exemple, les courbes sont enregistrées pour différentes valeurs d'élasticité de l'échantillon pour une valeur fixe de la raideur de la sonde AFM (figure 12) et des paramètres de l'interaction. On constate que la pente de la courbe dans la partie répulsive de l'interaction diminue au fur et à mesure que l'élasticité de l'échantillon devient faible devant celle du levier. Cela montre clairement que l'interaction agit sur le levier mais aussi sur l'échantillon et peut donc déformer plus l'échantillon que le levier pour une même force (c'est le ressort le plus souple qui se déforme le plus sous l'effet d'une force...). Les étudiants prennent ainsi conscience que le choix de la valeur de la constante de raideur de la sonde AFM joue un rôle déterminant dans la caractérisation des propriétées viscoélastiques ainsi que sur les conditions d'imagerie d'échantillons souples en mode contact.

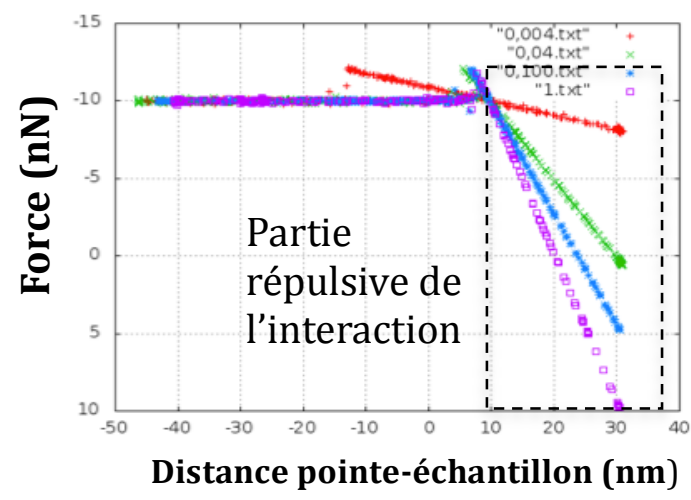

Figure 12: Courbes de force en fonction de la distance pointe-surface pour quatre valeurs différentes d'élasticité de l'échantillon $(0.004 ; 0.04$; $0.1 ; 1 \mathrm{~N} / \mathrm{m})$. La constante de raideur de la sonde AFM est fixée à $0,04 \mathrm{~N} / \mathrm{m}$ et les paramètres de l'interaction sont fixes.

Ils expérimentent aussi l'impact de la présence de force à longue distance telle que la force électrostatique (terme en $\mathrm{V}^{2}$ ) sur l'allure des courbes de spectroscopie de force. Cette étude illustre la différence entre les force dites à longues portées de celles à courtes portées. Cette différence est au cœur du fonctionnement des microscopies à force électrostatique [20] ou magnétique qui sont des techniques dérivées de l'AFM et qui sont très utiles pour la caractérisation des composants en nanoélectronique.

\subsection{Analyse in cellula par microscopie à épi-fluorescence de l'adhérence et du squelette de cellules oséoblastiques de souris structurées sur un patron adhésif de fibronectine (TP $\left.{ }^{\circ} 2\right)$}

Sur le site du Centre Universitaire de Biologie Expérimentale [21], les étudiants font connaissance avec une salle de culture de cellules. Chaque binôme dispose d'une hotte à flux laminaire sous laquelle ils pourront manipuler de manière stérile les cellules qui leur sont proposées. Les cellules ont été cultivées au préalable et sont stockées à $37^{\circ} \mathrm{C}$ dans un incubateur à $\mathrm{CO}_{2}$ sous atmosphère humide. Chaque étudiant dispose d'une boite contenant environs $5 \times 10^{4}$ cellules. Il s'agit d'une lignée de cellules ostéoblastiques de souris, ces cellules ont été génétiquement modifiées pour exprimer l'intégrine Béta3 couplée à une protéine de fusion fluorescente verte (GFP). L'intégrine $\beta 3$ est une protéine qui traverse la membrane plasmique des cellules animales et qui se regroupe en amas sur les sites d'adhésion que développent les cellules sur leur substrat (figure13). Au cours des 4 heures du TP n², les 
étudiants devront préparer 2 échantillons, l'un sera utilisé pour la caractérisation en microscopie à épi-fluorescence et l'autre pour la caractérisation en microscopie à Force Atomique (AFM). Dans ce but les étudiants doivent détacher les cellules de leur support, les dénombrer exactement et les ensemencer sur un patron adhésif de fibronectine (lamelle CYTOOstarter). Après 3 heures d'adhérence sur leur support, les échantillons sont fixées puis sont soit conservés dans un tampon physiologique pour être observées en AFM, soit montés entre lame et lamelle pour être observés par microscopie à épi-fluorescence.

La numération cellulaire est la détermination du nombre de cellules contenues dans un volume précis de milieu liquide. Celle-ci est importante à définir pour ensemencer précisément la lamelle micro patronnées de fibronectine. On exprime le résultat de la numération en concentration cellulaire, c'est à dire en nombre de cellules par litre. La numération cellulaire est réalisée directement par comptage au microscope inversé à contraste de phase, à l'aide d'une lame de comptage spéciale (ou cellule de numération de Malassez, figure 12).
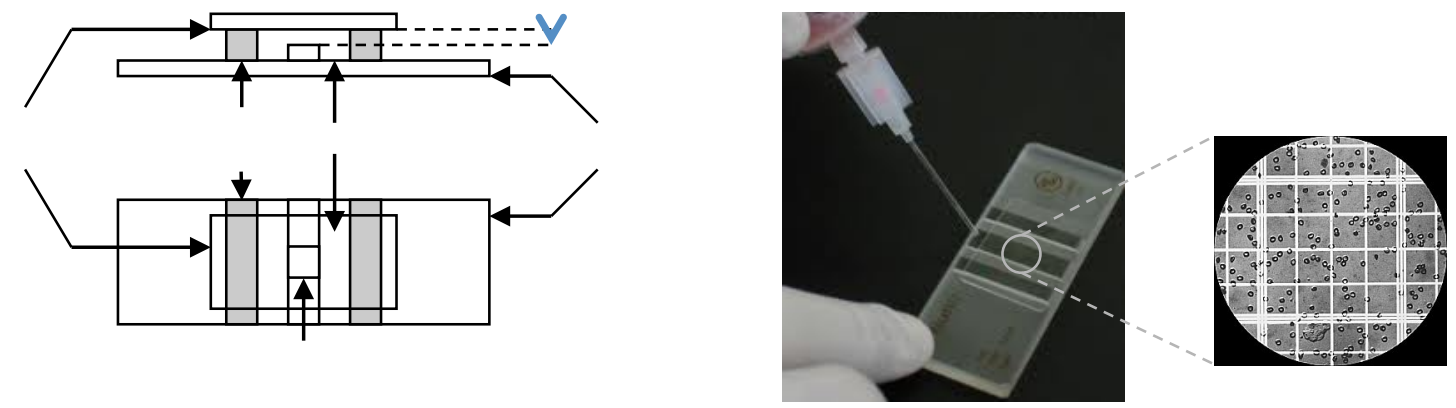

Figure 13: Une cellule de numération est une lame porte objet dans laquelle est creusée une chambre de comptage de volume connu. C'est une lame épaisse en verre, comportant des rigoles et un quadrillage. La cellule de numération de Malassez possède un quadrillage spécifique comportant 100 rectangles : parmi les 100 rectangles totaux, on trouve 25 rectangles qui sont divisés en 20 petits carrés afin de faciliter le comptage. Le volume correspondant au quadrillage total est égal à $1 \mathrm{~mm}^{3}=10^{-6} \mathrm{dm}^{3}(1 \mu \mathrm{L})$.

Les étudiants détecteront i) l'intégrine $\beta 3$ (en fluorescence verte) amassées en des sites particuliers sur la face ventrale adhésive des cellules, ii) le squelette d'actine (en fluorescence rouge) et iii) le noyau cellulaire (en fluorescence bleue) (figure 14). La fluorescence du squelette d'actine a été obtenue par ajout dans le fixateur-perméabilisant (Paraformaldehyde 4\%-Triton X100 0,3\%) d'une toxine très spécifique du polymère d'actine (la phalloïdine) couplée à un fluorochrome TRITC. La fluorescence du noyau a été obtenue par l'ajout d'un intercalant de l'ADN (le DAPI) dans le milieu de montage (Mowiol) qui fluoresce en bleu sous une excitation UV. Les microscopes à épi-fluorescence sont équipés d'une caméra et piloté par le logicel AxioVision (carl zeiss MicroImaging), les images obtenues sont sauvées sous un format .tif et .zvi avant d'être analysées (fig 13).

L'analyse qualitative et quantitative du cytosquelette d'actine et des adhérences $\beta 3$ est menée à la maison à l'aide d'un logiciel d'image (Fiji, scientific image processing) disponible gratuitement sur internet [22]. Au cours de cette séance les étudiants qualifient et quantifient les structures sub-cellulaires à l'échelle micrométrique, les mêmes structures seront abordées à l'échelle nanométrique par l'exploration AFM de l'échantillon (TP n³).

$\mathrm{Au}$ cours de cette séance, certains étudiants découvrent le vivant, les cellules et les outils pour les manipuler et les observer (micropipettes, microscope optique). C'est avec enthousiasme qu'ils s'attèlent à la tache, au bout des 4 heures que dure le TP ils sont capables de définir une 
cellule animale et d'en donner les échelles de tailles (de la cellule entière aux structures subcellulaires).

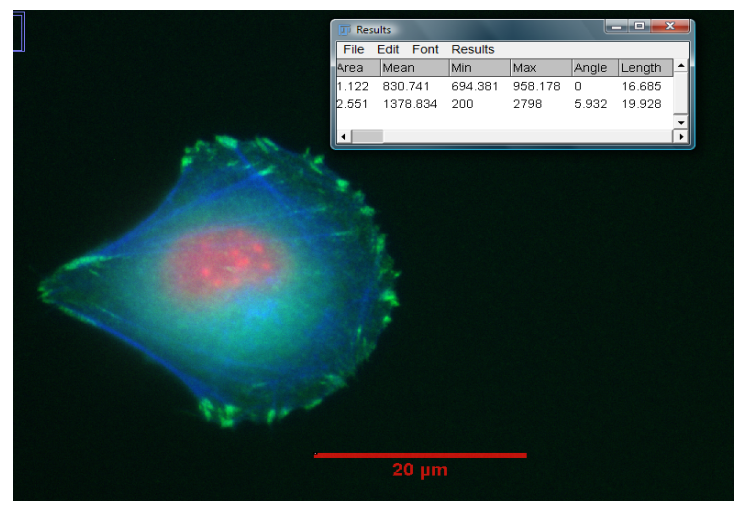

Figure 14 : Observation des marquages fluorescents (vert, rouge et bleu) grâce à un microscope à épi-fluorescence équipé d'un objectif $63 \mathrm{X}$ à immersion dans l'huile et muni des différents filtres appropriés. Le triple marquage révèle l'organisation et la localisation des intégrines $\beta 3$ (fausses couleurs vertes), des polymères d'actine (fausse couleur bleue) et de l'ADN dans le noyau (fausse couleur rouge), d'une cellule adhérente sur un patron adhésif de Fibronectine en forme d'arbalète (CYTOOchips ${ }^{\mathrm{TM}}$ ). Image obtenue en TP et issue de CR d'étudiants.

\subsection{Etude comparée par AFM des propriétés morphologiques et mécaniques de cellules ostéoblastiques de souris adhérentes sur un patron adhésif de fibronectine (TP $\left.n^{\circ} 3\right)$}

L'objectif de cette dernière séance de TP est double :

(1) Etudier en milieu aérien (à l'air) la morphologie à l'échelle micronique et nanométrique des cellules ostéoblastiques de souris qui ont adhéré et se sont étalées sur des substrats micro-bio-fonctionnalisés lors du TP précédent.

(2) Caractériser qualitativement les propriétés viscoélastiques d'une cellule en son centre puis surses bords.

Les caractérisations AFM viendront compléter les caractérisations obtenues en microscopie à épi-fluorescence sur les cellules. Au cours de cette séance, les étudiants devront mettre à profit les démarches expérimentales utilisées au cours des deux séances précédentes.

\subsubsection{Caractérisation morphologique de cellule par AFM}

La caractérisation morphologique par AFM des cellules ostéoblastiques de souris fixées sur le substrat CYTOOchips ${ }^{\mathrm{TM}}$ nécessite avant de les déshydrater d'éliminer tous les sels présents dans le tampon physiologique où baignent encore les cellules fixées au paraformaldehyde $4 \%$. L'échantillon est donc rincé dans un bain d'eau dé-ionisée puis laissé séché à l'air. Pendant ce temps et avec l'aide de l'enseignant (ou du technicien), une sonde AFM est placée sur la tête de l'AFM. Puis la lamelle CYTOOchips ${ }^{\mathrm{TM}}$ est mise en place sur la platine AFM équipée d'une optique (objectif 40x) qui permet de repérer la zone où la pointe sera engagée afin d'observer une cellule unique.

Dans un premier temps la cellule est imagée dans sa totalité afin de mesurer ses dimensions globales et d'identifier ses différentes parties (figure 15a); les étudiants font alors le lien avec les images optiques obtenues en fluorescence. La microscopie optique à épi-fluorescence révélait les adhérences cellulaires à l'extrémité du squelette tendu que forme les microfilaments d'actine, les étudiants découvrent grâce à l'imagerie AFM les contours membranaires sous tendu par le squelette d'actine ainsi que la zone patronnée en Fbn (figure 
15b). D'autres structures cellulaires non ou peu résolues au microscope optique apparaissent clairement en imagerie AFM : les filopodes et les lamellipodes. Ces extensions membranaires protruent aux bords cellulaires grâce à la polymérisation des filaments d'actine sous jacents.

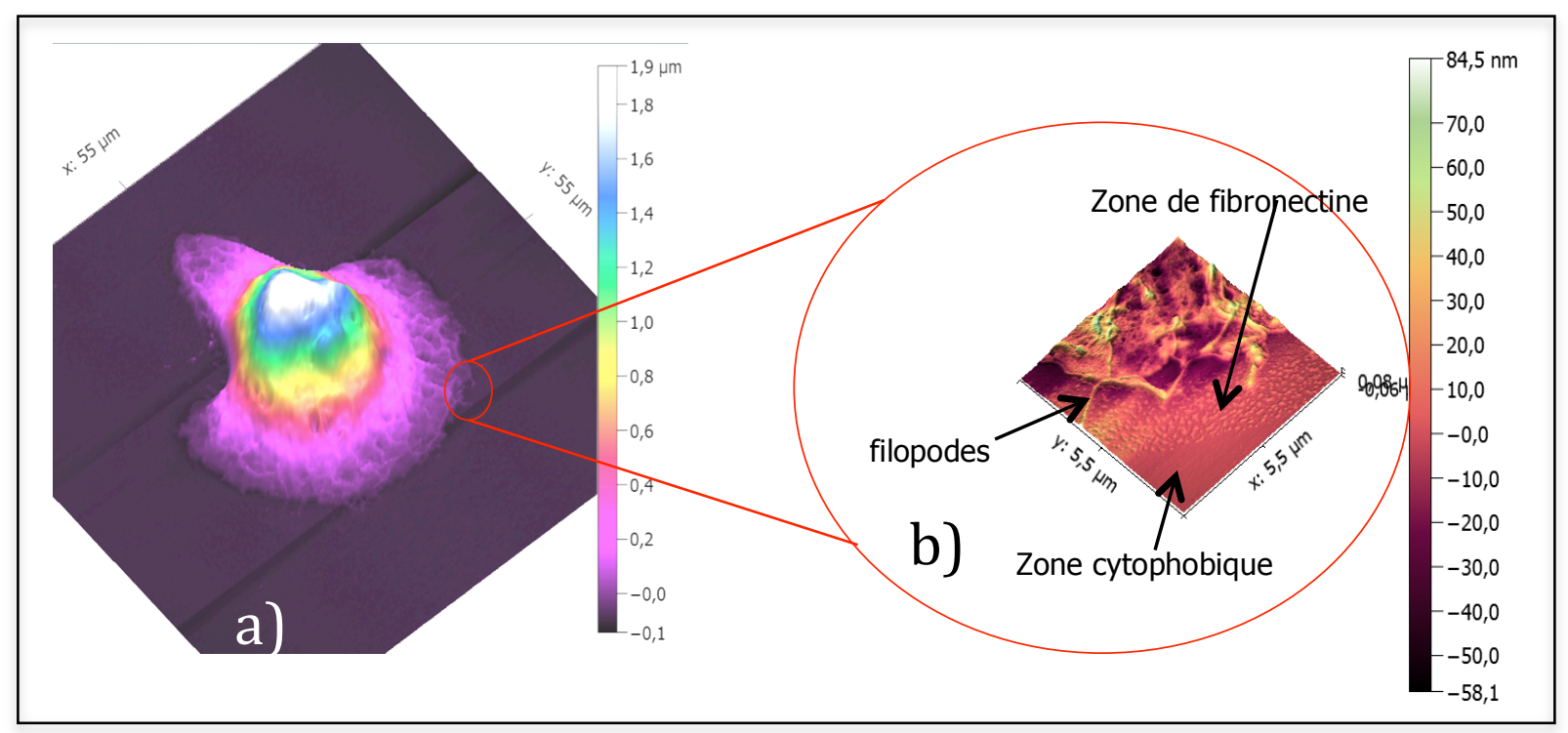

Figure 15 : a) image AFM en 3D d'une cellule biologique ancrée sur un motif de Fibronectine en forme d'arbalète ; b)Image AFM sur le bord de la cellule qui montre les filopodes, sorte de nano-antennes qui permettent à la cellule de sonder son environnement. Image obtenues en TP et issues de CR d'étudiants.

Le fait d'apercevoir encore le patron de Fibronectine bordant l'extension membranaire donne aux étudiants une vision dynamique d'une cellule fixée. La cellule n'a pas réalisé complètement son étalement ; elle est encore en cours d'exploration de son environnement. Les filopodes sont les extensions membranaires que la cellule projette vers l'extérieur afin d'explorer (sonder) son environnement immédiat (ces extension membranaires sont « tubées » grâce à la polymérisation des microfilaments d'actine qui s'organisent parallèlement les uns aux autres). Ces nano sondes se stabilisent sur le support adhésif et se déploient en lamellipodes grâce à la polymérisation d'un réseau branché de microfilaments d'actine (figure 16).
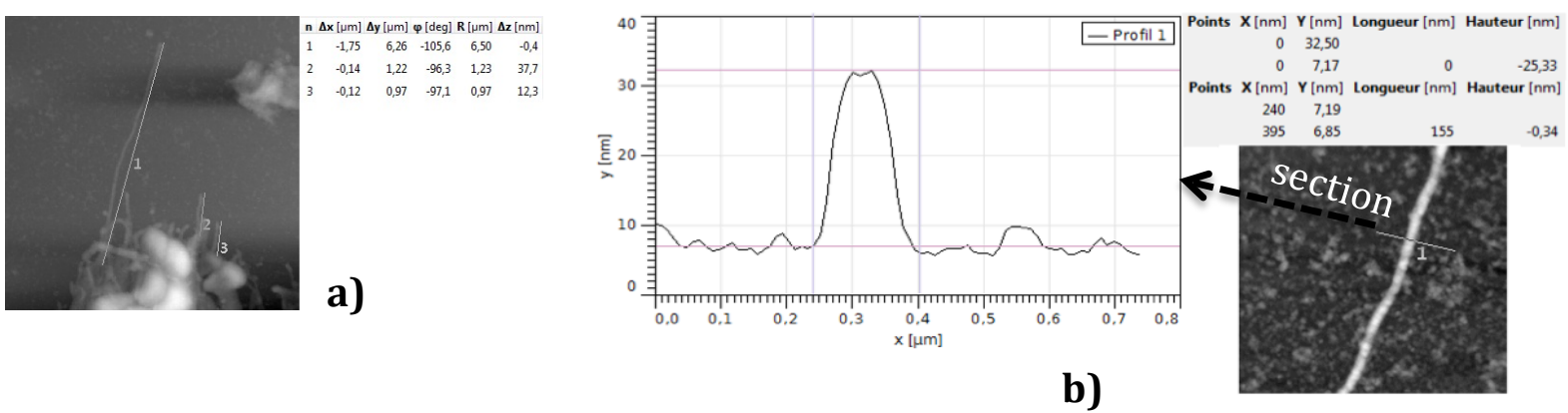

Figure 16 : Analyse en mode Tapping des projections cellulaires les plus fines : les filopodes. Quantification des longueurs (a) et du diamètre (b) caractéristiques. Les filopodes sont composés essentiellement de filaments d'actine orientés parallèlement les uns avec les autres. Image et section obtenues en TP et issues de CR d'étudiants.

Une fois que les étudiants ont terminé d'identifier différents composants d'une cellule ostéoblastique de souris, débute alors la deuxième partie du TP consacrée à la caractérisation qualitative des propriétés viscoélastiques. Cette deuxième partie fait appel aux savoir-faire et connaissance acquises sur la spectroscopie de force lors du premier TP de cette série de nanobiophysique. 


\subsubsection{Etude de la réponse mécanique de la cellule}

La sonde AFM peut servir comme sonde morphologique mais aussi comme sonde locale de force grâce au mode spectroscopie et ainsi comparer les réponses mécaniques de différents matériaux ou différentes zones d'un échantillon.

L'objectif de cette partie est donc de comparer la déformation élastique subit par levier en fonction de la distance pointe-échantillon entre trois zones : sur le substrat de verre, au centre de la cellule puis au bord de la cellule. La figure 17 montre une image de cellule (et les trois courbes de force associées.

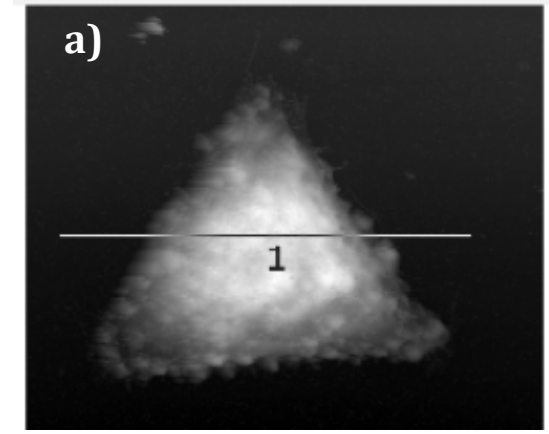

c)

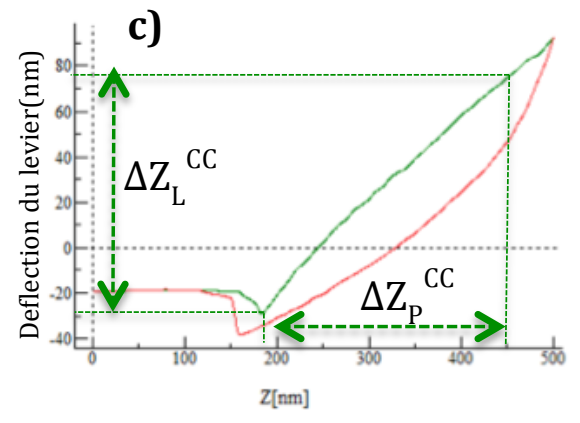

b)

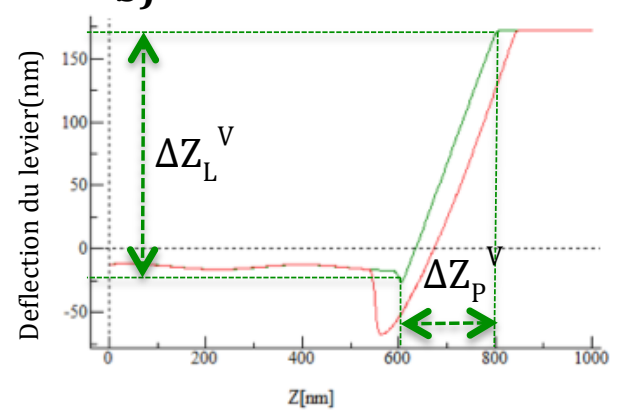

d)

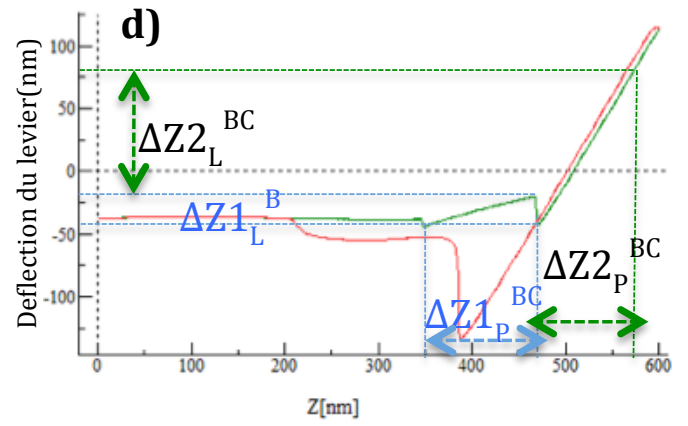

Figure 17: a) image topographique d'une cellule fixée sur un motif de forme $Y$, barre latérale représente $40 \mu \mathrm{m}$, l'échelle en $Z$ est de 0 à $1.5 \mu \mathrm{m}$ (noir au blanc); b) courbe de force obtenue sur le substrat de verre, c) courbe obtenue au centre de la cellule et d) courbe obtenue sur un bord de la cellule. Les courbes vertes sont obtenues lors de la phase d'approche de la pointe alors que les courbes rouges le sont lors de la phase de retrait. Image et courbes obtenues en TP et issues de CR d'étudiants.

Afin d'obtenir la mesure de la déflection du levier en nanomètre, les étudiants effectuent la calibration de la photodiode sur un échantillon de silicium considéré comme infiniment rigide devant la raideur du levier AFM.

Pour comparer l'élasticité relative des trois zones, les étudiants calculent la ou les pente(s) des courbes de force lors de l'approche (courbes vertes) qui correspond au rapport $P$ entre la déformation du levier AFM et l'élongation du scanner piézoélectrique, à savoir $P=\Delta Z_{L}^{I} / \Delta Z_{P}^{I}$. Lorsque la valeur du rapport s'approche de 1 cela signifie que la déformation du levier est égale à l'élongation du scanner et donc que toute la déformation est subie par le levier. Si le rapport $\mathrm{P}$ est inférieur à 1 cela signifie que l'échantillon subit une déformation et donc que l'échantillon a une élasticité qui ne peut plus être considérée comme beaucoup plus élevée que celle du levier. Le tableau 1 résume le calcul de ces pentes.

\begin{tabular}{|c|c|c|c|}
\hline Zone & verre & $\begin{array}{c}\text { Centre de la } \\
\text { cellule }\end{array}$ & Bord de la cellule \\
\hline Pente $=\Delta Z_{\mathrm{L}}{ }^{\mathrm{I}} / \Delta \mathrm{Z}_{\mathrm{P}}{ }^{\mathrm{I}}$ & $\mathbf{2 0 0} / \mathbf{2 0 0}=\mathbf{1}$ & $\mathbf{1 0 0 / 2 6 0 = 0 , 3 8}$ & $\mathbf{P 1 = 2 5 / 1 2 5 = 0 , 2} \quad \mathbf{P 2 : 1 0 0 / 1 0 0 = 1}$ \\
\hline
\end{tabular}

Tableau 1 Valeur des pentes de la courbe de force à l'approche sur trois zones différentes de l'échantillon. 
On constate que sur le verre la pente égale 1 ce qui est logique car le substrat de verre est très rigide comparée à la raideur du levier AFM. Par contre au centre de la cellule, le rapport est égal à 0,38 ce qui montre que son élasticité est plus faible que celle du levier AFM. La courbe obtenue sur le bord de la cellule montre un comportement plus complexe avec la présence de deux pentes, la première $\mathrm{P} 1$ égale à 0,2 et la deuxième $\mathrm{P} 2$ égale à 1 . Ce comportement a été enregistré sur plusieurs cycles de courbe d'approche-retrait et fut reproductible. A la suite de ce cycle, une image AFM a été réalisée et aucune dégradation de la surface de la cellule observée. Une interprétation possible de la présence de ces deux pentes est que la sonde AFM interagit d'abord avec la membrane qui est souple, puis une fois que la membrane est déformée de telle sorte qu'elle touche la surface de verre, le comportement du levier est alors équivalent à celui observé sur le substrat de verre.

Ainsi au vu de ces résultats les étudiants peuvent classer la rigidité $R$ des différentes zones étudiées et classifiées: $R_{\text {verre }}>R_{\text {centre de la cellule }}>R_{\text {bord de la cellule. Cette classification concorde avec }}$ des résultats de recherche récents[23].

La technique de spectroscopie de force est à la base de la méthodologie utilisée par les biomécanicien de la cellule pour estimer le module de Young des cellules [24], cette partie de $\mathrm{TP}$ constitue donc une initiation à cette approche expérimentale plus complexe (une sonde colloïdale et un logiciel de traitement des données spécifique sont alors requis).

\section{Retour d'expérience sur le plan pédagogique et impact sur l'orientation des étudiants}

Cette unité d'enseignement étant fortement orientée vers l'expérimentation, l'évaluation des étudiants est réalisée comme suit : $50 \%$ de la note finale correspond à la moyenne des notes de TP obtenue sur la base des comptes rendu de TP. A la fin de l'enseignement (cours et TP) un examen écrit théorique (50\% de la note finale) comportant 3 parties distinctes (nanochimie, nanophysique et nanobiophysique) permet de contrôler les acquis théoriques des étudiants. Cette évaluation met donc fortement l'accent sur les aptitudes pratiques développées durant cet enseignement.

Afin d'évaluer la perception de cette UE par les étudiants et son impact dans leur orientation en M2 ainsi que son utilité, nous avons demandé leur avis aux étudiants. Une enquête a été menée auprès des étudiants ayant suivi le master 1 Nano en 2011 et 2012, dix huit étudiants au total ont répondu au questionnaire de manière anonyme. Le questionnaire se compose de quatre ou cinq questions auxquelles les étudiants devaient répondre en cochant oui ou non:

Q1 : Cette UE a pour objectif d'illustrer la pluridisciplinarité des Nanosciences, pensez vous que cet objectif est atteint?

Q2 : a) Cette UE étant par essence pluridisciplinaire a donc nécessité l'intervention de plusieurs enseignants, est-ce un point positif pour votre formation?

b) l'intervention des différents intervenants vous paraît elle cohérente ?

Q3: Est ce que les thématiques abordées dans les TP illustrent bien l'enseignement théorique reçu dans votre formation?

Q4 : a) Avez-vous apprécié l'aspect inter-plateformes et couplé des deux séries de TP (Nanobiophysique et Nanochimie/nano-caractérisation)?

b) Auriez-vous préféré des TP indépendants des uns des autres?

Q5 destinée au M2: a) Est ce que cette UE vous a servi pour votre choix d'orientation?

b) Avez vous utilisé ces méthodes de caractérisation ou d'élaboration dans la suite de votre formation (Master 2) ou lors de vos stages? 
Un espace « commentaire libre » était disponible pour chaque question afin que les étudiants qui le souhaitaient, puissent s'exprimer plus en détails.

Les quatre premières questions qui portent sur les aspects pédagogiques de cette UE ont été soumises à tous les étudiants. La dernière question portant sur l'impact de cette UE sur le choix d'orientation en Master 2 (Nanophysique, Nanochimie, Nanobiotechnologies en Nanoingéniérie) ainsi que sur l'utilité de l'apprentissage des méthodes expérimentales pour leurs stages (stages de deux ou trois mois en M1 et de quatre à six mois en M2), ont été uniquement posées aux étudiants actuellement en deuxième année de master Nano.

L'analyse des quatre premières questions est compilée dans la figure 18 sous forme de pourcentage.

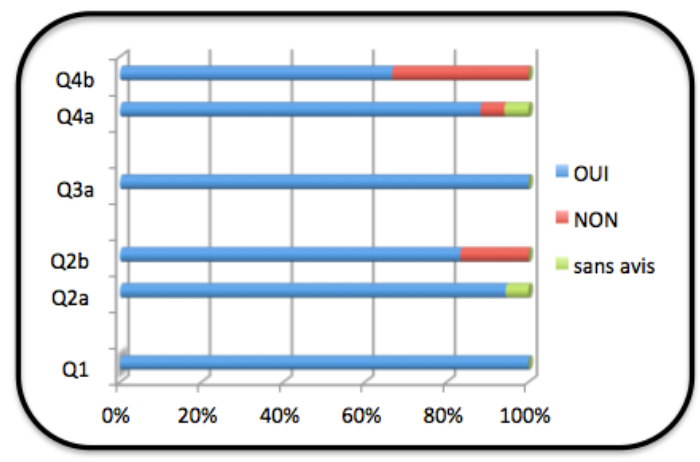

Figure 18: diagramme statistique illustrant la perception de l'UE par les étudiants

On constate sur ce graphique que les étudiants sont unanimes (100\% de oui à la question 1) pour dire que cette UE illustre bien la pluridisciplinarité des Nanosciences. La lecture des commentaires libres permet de mieux comprendre pourquoi : "Avoir plusieurs enseignants était intéressant pour pouvoir avoir plusieurs manières d'expliquer» ou encore "Grâce à plusieurs interventions de différents professeurs nous avons bien pu nous rendre compte des différentes applications possibles des nanosciences».

A travers la question 2a) l'intervention de trois enseignants-chercheurs issus de trois disciplines différentes est jugée comme un point positif à $95 \%$ ce qui doit surement être lié à la bonne cohérence de leurs interventions respectives (question $2 \mathrm{~b}, 80 \%$ la juge cohérente).

Le lien entre enseignement théorique et expérimental se révèle aussi très bien apprécié par tous les étudiants (question 3) et les commentaires libres soulignent même leur présence comme essentielle : «Je pense que ces TP, étaient nécessaires à la bonne compréhension de cet enseignement. Les TP suivaient parfaitement le programme et étaient réellement intéressant». Enfin, l'aspect inter-plateformes et couplé des deux séries de TP a été bien apprécié (Question 4a) même si certains étudiants auraient préféré des TP complètement indépendants des uns des autres. Cette relative contradiction s'explique surement par le fait qu'ils devaient rendre un seul CR par série de TP et non pas un CR par TP, les obligeant ainsi à faire un effort de synthèse plus important.

L'analyse de ces réponses tend à montrer que les enseignants ont réussi à construire une approche pédagogique cohérente entre les différentes disciplines abordées dans cette UE et adaptée à l'auditoire provenant de licences différentes possédant donc des «backgrounds » non identiques. Cette nécessité se retrouve très souvent dans les masters dédiés à des domaines par essences pluridisciplinaires comme la microélectronique [25] ou le traitement des déchets nucléaires.

On peut donc conclure que les quelques heures accordées à chaque enseignant pour la mise en place de cette UE à fort caractère expérimental, par l'UFR PHITEM ont porté leurs fruits pour assurer la cohérence pédagogique de cette UE pluridisciplinaire. 
La synthèse des réponses à la question 5 évaluant d'une part l'impact de cette UE dans le choix d'orientation en M2 et d'autre part l'utilité de ces techniques dans les stages, est illustrée dans la figure 19.

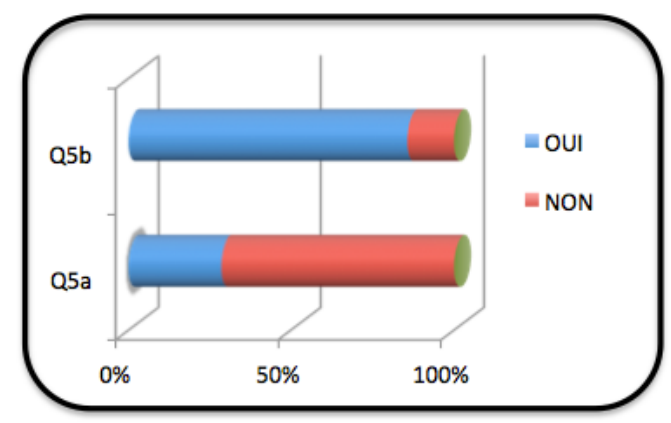

Figure 19: diagramme statistique des réponses à la question 5 portant sur l'impact de l'UE dans le choix d'orientation en M2 et sur l'utilité de l'apprentissage des techniques «Nano» pour les stages.

A travers les réponses à la question $5 \mathrm{a}$, on constate que cette UE a un impact faible sur le choix d'orientation dans les mentions (nanophysique, nanochimie, nanobiotechnologie ou nanoingéniérie) proposé en M2 Nano. Cela montre que la majorité des étudiants qui s'engage en master 1 Nano ont déjà une idée bien précise sur leur poursuite d'étude. Néanmoins il faut noter que la lecture des commentaires libres montre que pour certains étudiants non encore fixés sur leur choix de mention en M2 Nano, cette UE a un impact certain : "C'est une UE très importante qui permet de s'orienter dans son choix des M2 disponibles».

Enfin concernant l'impact de l'apprentissage de nombreuses techniques d'élaboration et de caractérisation à l'échelle nanométrique au cours des stages, la question $5 \mathrm{~b}$ montre clairement que la plupart des étudiants ont été amenés à utiliser certaines d'entre-elles au cours de leurs stages. Ces savoir-faire acquis durant cette UE se révèlent donc très utiles au cours de leur première expérience dans le monde professionnel. De plus la lecture des commentaires libres soulignent aussi l'intérêt de l'aspect couplé des TP dans l'apprentissage des démarches scientifiques dans le domaine de la recherche : "Le TP inter-plateformes était vraiment bien. $C$ 'est à mon avis très pertinent d'avoir l'aspect fabrication (fonctionnalisation, etching...) lors d'un premier TP et de pouvoir caractériser ces manip lors d'un deuxième TP car c'est très formateur pour les expériences menées en stage».

\section{Conclusions et Perspectives}

A travers la série de TP «Nanoparticules» qui est un thème emblématique des Nanosciences, les étudiants ont pu réaliser l'élaboration de nanoparticules métalliques par des méthodes de synthèse chimiques bien établies. Ils ont pu ensuite caractériser leurs propriétés dimensionnelles par spectroscopique optique technique bien connue des chimistes et compléter ces caractérisations grâce au microscopie MEB et AFM. Grâce à l'AFM en mode nanomanipulation, l'adhérence de ces nanoparticules métalliques sur une surface de graphite a pu être menée et donc les notions de physisorption versus chimiosorption abordées.

$\mathrm{Au}$ cours de la série de TP de Nanobiophysique, les étudiants ont pu observer par microscopies optique et à force atomique un substrat micro-bio-fonctionnalisée dont la fabrication relève des techniques de dépôts de la microélectronique. Ils ont ensuite suivi un protocole biologique spécifique pour présenter des cellules animales sur un substrat microbio-fonctionnalisé qu'ils ont ensuite pu caractériser par microscopies de fluorescence puis atomique. La complémentarité des deux techniques pour observer différents constituants d'une cellule a naturellement émergé. Enfin ils se sont initiés à la nanobiomécanique grâce à l'étude qualitative des propriétés viscoélastiques de parties distinctes de la cellule sondées via le mode de spectroscopie de force de l'AFM. 
La perception de cette UE par des étudiants et l'identification des points forts et faibles ont été obtenues grâce à une enquête anonyme auprès des étudiants sous la forme d'un questionnaire. Les principaux résultats de l'enquête montrent que ces deux séries de TP apparaissent comme indispensables aux étudiants pour rendre concret l'aspect pluridisciplinaire des nanosciences. L'intervention de plusieurs enseignants est ressentie comme une richesse car elle est bien cohérente. Cette UE « Nanosciences » apparaît comme une aide dans le choix d'orientation en M2 Nano pour les étudiants indécis. Les techniques de caractérisation utilisées en TP semblent bien correspondre au monde académique et industriel puisse qu'une majorité des étudiants indiquent y avoir eu recours durant leurs stages en laboratoire ou en entreprise.

$\mathrm{Au}$ niveau pratique, il est clair que le développement de ces TP Nano interdisciplinaires repose en grande partie sur la synergie entre des plateformes de formation de très haut niveau qui sont équipées d'instruments à la pointe en micro/nanotechnologies.

Du point de vue des enseignants-chercheurs de chimie, biologie et physique spécialistes en nanosciences et nanotechnologies qui interviennent dans cette UE, la mise en place de ces deux séries de TP a constitué une opportunité unique d'échanger d'un point de vue pédagogique mais aussi scientifique sur leurs approches respectives de la thématique «Nano» dans les différentes disciplines.

Dans l'avenir, les perspectives de ces séries de TP sont multiples et se situent à plus ou moins long terme:

(1) à court terme par leur adaptation au format d'une journée pour être proposée à l'école européenne ESONN dès septembre 2013,

(2) à moyen terme par la formation d'enseignants-chercheurs du réseau CNFM afin d'en faire profiter le plus grand nombre de formation,

(3) à un peu plus long terme, les adapter pour les proposer aux formations de Master en filière biologie afin d'attirer plus d'étudiants biologistes dans le Master de Nanobiotechnologie,

(4) proposer une version allégée pour les lycéens à travers le programme grenoblois Nano@school [26] qui leur est dédié afin d'illustrer la complémentarité des sciences physique et chimique avec les sciences de la vie et de la terre (SVT).

\section{Remerciements}

Les auteurs remercient l'ensemble des personnels techniques du CUBE, du plateau de chimie et du CIME-Nanotech pour leur support dans le montage de ces nouveaux TP.

Les auteurs remercient l'UJF pour son support financier de 10kE attribué via Bonus Qualité Formation en 2010 au titre de «l'innovation pédagogique pour une plus grande qualité de services rendus aux étudiants et aux enseignants $\gg$.

Les auteurs remercient l'UFR PhITEM pour l'attribution de quelques heures de TD par enseignant pour la mise en place de ces TP.

\section{Bibliographie}

[ 1 ] Site web du Master N2: http://physique-eea.ujf-grenoble.fr/MasterNano/ et http://www.ujf-grenoble.fr/formation/diplomes/masters/domaine-sciences-technologiessante/masters-mention-nanosciences-nanotechnologies-217092.htm?RH=1314955048444

[2] www.cime.inpg.fr/

[3] http://cube.ujf-grenoble.fr/

[4] «Gold nanoparticles: assembly and electrical properties in 1-3 dimensions», G. Smith \& U. Simon, Chem. Commun., 2005, 6, 697-710. 
[ 5 ] «Gold nanoparticles: assembly, supramolecular chemistry, quantum-size-related properties, and applications toward biology, catalysis, and nanotechnology», M-C. Daniel, D. Astruc, Chem. Rev., 2004, 104, 293-346.

[6] « Recent advances in nanotechnology applied to biosensors », X. Zhang, Q. Guo, D. Cui, Sensors, 2009, 9, 1033-1053.

[7] « Coagulation of colloidal gold », B.V. Enüstün and J. Turkevich, J. Am. Chem. Soc., 1963, 85 (21), pp 3317-3328.

[8] « Size control over spherical silver nanoparticles by ascorbic acid reduction », Y.Qin et al. Colloids and surfaces A : Physicochem. Eng. Aspects, 2010, 372, 172-176.

[9] Site web de la plateforme PCA : http://pca.ujf-grenoble.fr/

[10] « Nanomechanical analysis of cells from cancer patients", S. E. Cross, Y.S. Jin, J. Rao, J.K. Gimzewski, Nature Nanotechnology, 2007, 2, 780.

[11] «L'embryon sous l'emprise des gènes et de la pression », Farge, E., Pour la Science, 2009, vol. 379, pp. 42-49.

[12] « Mechanical induction in embryonic development and tumor growth integrative cues through molecular to multicellular interplay and evolutionary perspectives ", FernandezSanchez, M., E., Serman, F., Ahmadi, P., Farge, E., Methods Cell Biol., 2010, vol. 98, pp. 295-321.

[13] « Autonomous micro-magnet based systems for highly efficient magnetic separation », L. F. Zanini, N. M. Dempsey, D. Givord, G. Reyne, and F. Dumas-Bouchiat, Appl. Phys. Lett., 2011, 99, 232504.

[14] " Actin machinery and mechanosensitivity in invadopodia, podosomes and focal adhesions ", Albiges-Rizo, C., O. Destaing, B. Fourcade, E. Planus \& M. R. Block, J Cell Sci, 2009, 122, 3037-49.

[15] « Environmental sensing through focal adhesions », Geiger, B., J. P. Spatz \& A. D. Bershadsky, Nat Rev Mol Cell Biol, 2009, 10, 21-33.

[16] http://www.cytoo.com/

[17] «Fabrication of hierarchical micro-nanotopographies for cell attachment studies », M J Lopez-Bosque et al, Nanotechnology, 2013, 24, 255305.

[18] « An augmented reality nanomanipulator for learning nanophysics : the "Nanolearner" platform », F. Marchi, S. Marlère, J.L. Florens, A. Luciani and J. Chevrier, LNCS 6250, Journal of Transactions on Edutainment IV, Springer 2010, p157-175.

[19] «Le concept du NanoLearner : Les mains dans le Nanomonde de l'Université vers le grand public », F. Marchi, J. Castet, S. Marlière, N. Castagné, J. Chevrier, A. Luciani et JL. Florens, revue électronique J3eA, 2010, Volume 9 http://dx.doi.org/10.1051/j3ea/2010017.

[20] « Characterisation of trapped electric charge carriers behavior at nanometer scale by electrostatic force microscopy », F. Marchi, R. Dianoux, H.J.H Smilde, P. Mur, F. Comin and J. Chevrier, Journal of Electrostatics, 2008, pp538-547.

[21] Site web de la plateforme du CUBE : http://www-biologie.ujf-grenoble.fr/CUBE/

[22] http://fiji.sc/wiki/index.php/Downloads

[23] «Gradient of rigidity in the lamellipodia of migrating cells revealed by atomic force microscopy.» Laurent VM, Kasas S, Yersin A, Schäffer TE, Catsicas S, Dietler G, Verkhovsky AB, Meister JJ. Biophys J. 2005 Jul;89(1):667-75. Epub 2005 Apr 22.

[24] "Atomic force microscopy probing of cell elasticity», T.G. Kuznetsova, M.N. Starodubtseva, N. I. Yegorenkov, S.A. Chizhik, R.I. Zhdanov, Micron, 2007, 38, 824 833. 
[25] « Master international double diplômant entre la France et la Chine sur la base du processus de Bologne », O. Bonnaud, L. Senhadji, H. Shu, L. Wei, revue électronique J3eA, Volume 10, 2011. DOI : http://dx.doi.org/10.1051/j3ea/2011012

[26] http://www.nanoatschool.org/ 